\title{
The Properties and Redshift Evolution of Intermediate-Luminosity Off-Nuclear X-ray Sources in the Chandra Deep Fields
}

\author{
B. D. Lehmer, ${ }^{1}$ W. N. Brandt, ${ }^{1}$ A. E. Hornschemeier, ${ }^{2}$ D. M. Alexander ${ }^{3}$ F. E. Bauer,${ }^{4}$ \\ A. M. Koekemoer, ${ }^{5}$ D. P. Schneider, ${ }^{1} \&$ A. T. Steffen ${ }^{1}$
}

\begin{abstract}
We analyze a population of intermediate-redshift $(z \approx 0.05-0.3)$, off-nuclear X-ray sources located within optically-bright galaxies in the Great Observatories Origins Deep Survey (GOODS) and Galaxy Evolution from Morphology and SEDs (GEMS) fields. A total of 24 off-nuclear source candidates are classified using deep Chandra exposures from the Chandra Deep Field-North, Chandra Deep Field-South, and Extended Chandra Deep Field-South; 15 of these are newly identified. These sources have average X-ray spectral shapes and optical environments similar to those observed for off-nuclear intermediate-luminosity $\left(L_{\mathrm{X}} \geq 10^{39} \mathrm{erg} \mathrm{s}^{-1}\right.$ in the $0.5-2.0 \mathrm{keV}$ band) X-ray objects (IXOs; sometimes referred to as ultraluminous X-ray sources [ULXs]) in the local universe. This sample improves the available source statistics for intermediate-redshift, off-nuclear sources with $L_{\mathrm{X}} \gtrsim 10^{39.5} \mathrm{erg} \mathrm{s}^{-1}$, and it places significant new constraints on the redshift evolution of the off-nuclear source frequency in field galaxies. The fraction of intermediate-redshift field galaxies containing an off-nuclear source with $L_{\mathrm{X}} \gtrsim 10^{39} \mathrm{erg} \mathrm{s}^{-1}$ is suggestively elevated ( $\approx 80 \%$ confidence level) with respect to that observed for IXOs in the local universe; we calculate this elevation to be a factor of $\approx 1.9_{-1.3}^{+1.4}$. A rise in this fraction is plausibly expected as a consequence of the observed increase in global star-formation density with redshift, and our results are consistent with the expected magnitude of the rise in this fraction.
\end{abstract}

\footnotetext{
${ }^{1}$ Department of Astronomy \& Astrophysics, 525 Davey Lab, The Pennsylvania State University, University Park, PA 16802, USA

${ }^{2}$ Laboratory for X-ray Astrophysics, NASA Goddard Space Flight Center, Code 662, Greenbelt, MD 20771, USA

${ }^{3}$ Institute of Astronomy, Madingley Road, Cambridge, CB3 0HA, United Kingdom

${ }^{4}$ Columbia Astrophysics Laboratory, Columbia University, Pupin Labortories, 550 W. 120th St., Rm 1418, New York, NY 10027, USA

${ }^{5}$ Space Telescope Science Institute, 3700 San Martin Drive, Baltimore, MD 21218, USA
} 
Subject headings: cosmology: observations — diffuse radiation — surveys — $\mathrm{X}$-rays: galaxies - X-rays: general

\section{Introduction}

Intermediate-luminosity X-ray objects (IXOs) in the local universe are off-nuclear X-ray sources having 0.5-8.0 keV luminosities exceeding $\sim 10^{39} \mathrm{erg} \mathrm{s}^{-1}$ (e.g., Colbert \& Ptak 2002). X-ray sources making up the high-luminosity end (i.e., $\gtrsim 2 \times 10^{39} \mathrm{erg} \mathrm{s}^{-1}$ ) of the IXO population are commonly referred to as ultraluminous X-ray sources (ULXs); they are referred to as "ultraluminous" because their inferred isotropic luminosities exceed that expected for an $\approx 10 M_{\odot}$ black hole accreting at the Eddington limit. Investigations of this enigmatic population suggest that many IXOs may be intermediate-mass black holes (IMBHs) with masses exceeding $\approx 10 M_{\odot}$ (e.g., Colbert \& Miller 2004; Miller et al. 2004) and/or normal high mass X-ray binaries (HMXBs) anisotropically beaming X-rays into our line-of-sight (e.g., King et al. 2001). Recent observations indicate IXOs may be a natural high-luminosity extension of the HMXB X-ray luminosity function, suggesting that these sources trace relatively young stellar populations (e.g., Gilfanov et al. 2004). Indeed, analyses of the physical and environmental properties of IXOs have shown that the number and cumulative X-ray luminosities of IXOs are correlated with star-formation rate (e.g., Kilgard et al. 2002; Swartz et al. 2004). As a consequence of this, IXOs are more commonly observed in late-type spiral and irregular galaxies than in early-type ellipticals. Furthermore, investigations of X-ray populations in early-type galaxies find that luminous $\left(L_{\mathrm{X}} \gtrsim 2 \times 10^{39} \mathrm{erg} \mathrm{s}^{-1}\right)$ IXOs (i.e., ULXs) are generally absent (e.g., Irwin et al. 2003); however, exceptions have been noted (e.g., Loewenstein et al. 2005).

Recently, the fraction of galaxies in the local universe containing IXOs (as a function of X-ray luminosity) has been constrained statistically using ROSAT observations of a sample of 766 relatively nearby $(D<66.7 \mathrm{Mpc})$ galaxies (Ptak \& Colbert 2004; hereafter PC04) from the Third Reference Catalog of Bright Galaxies (RC3; de Vaucouleurs et al. 1991). PC04 report that, after correcting for expected background sources, $\approx 12 \%$ and $\approx 1 \%$ of all local RC3 spiral and irregular galaxies have one or more IXOs with 2-10 keV luminosities $L_{\mathrm{X}}>10^{39} \mathrm{erg} \mathrm{s}^{-1}$ and $L_{\mathrm{X}}>10^{40} \mathrm{erg} \mathrm{s}^{-1}$, respectively. Here, 2-10 keV luminosities were estimated from the $0.2-2.4 \mathrm{keV}$ flux with an assumed IXO power-law photon index of $\Gamma=1.7$.

At increasing redshifts, it is plausibly expected that the fraction of galaxies hosting IXOs will increase as a result of the observed rise in global star-formation density with redshift (e.g., Madau et al. 1998; Ghosh \& White 2001). Evidence for the global evolution of starformation activity has also been observed as an increase in the average X-ray luminosity from 
normal galaxies out to $z \approx 1$ (e.g., Hornschemeier et al. 2002); IXOs likely play an important role in this evolution. Thus far, low X-ray flux levels and limited angular resolution have restricted the study of relatively distant $(z \gtrsim 0.05 ; D \gtrsim 200 \mathrm{Mpc})$ IXOs. However, deep multiwavelength extragalactic surveys that combine the optical imaging capabilities of the Hubble Space Telescope (HST) and the sub-arcsecond X-ray imaging of the Chandra X-ray Observatory (Chandra) have made the detection and classification of intermediate-redshift $(z \approx 0.05-0.3$; lookback times of $\approx 0.7-3.4 \mathrm{Gyr})$ off-nuclear sources possible. Hornschemeier et al. (2004; hereafter H04) isolated and characterized ten off-nuclear source candidates in optically-bright field galaxies with redshifts in the range $z=0.04-0.23\left(z_{\text {median }} \approx 0.1\right)$. This investigation utilized HST observations with Advanced Camera for Surveys (ACS) filters $B_{435}, V_{606}, i_{775}$, and $z_{850}$ from the Great Observatories Origins Deep Survey (GOODS; Giavalisco et al. 2004) and deep Chandra observations coincident with these fields through the $\approx 2$ Ms Chandra Deep Field-North (CDF-N; Alexander et al. 2003) and $\approx 1$ Ms Chandra Deep Field-South (CDF-S; Giacconi et al. 2002) surveys. H04 found that the fraction of field galaxies with detectable off-nuclear X-ray sources $\left(L_{0.5-2.0 \mathrm{keV}} \gtrsim 10^{38.9} \mathrm{erg} \mathrm{s}^{-1}\right)$ at $z \approx 0.1$ is $36_{-15}^{+24 \%}$, suggestively larger than that observed for galaxies in the local universe. Moreover, due to an angular-resolution bias, this "observed" fraction was only considered to be a lower limit to the true fraction, which would include off-nuclear sources with offsets smaller than the Chandra positional error circles. Unfortunately, a study of the dependence of this fraction upon off-nuclear source X-ray luminosity fraction was not possible due to limited source statistics, most notably for sources with $0.5-2.0 \mathrm{keV}$ luminosities $L_{\mathrm{X}} \gtrsim 10^{39.5} \mathrm{erg} \mathrm{s}^{-1}$.

In this investigation, we estimate the true fraction of intermediate-redshift field galaxies hosting off-nuclear X-ray sources as a function of $0.5-2.0 \mathrm{keV}$ luminosity and compare it with that observed for local galaxies (from PC04). We improve the source statistics available for intermediate-redshift, off-nuclear sources by combining the multiwavelength data within the $\approx 2 \mathrm{Ms}$ CDF-N and $\approx 1 \mathrm{Ms}$ CDF-S with new HST and Chandra observations of the Extended Chandra Deep Field-South (E-CDF-S; Lehmer et al. 2005a). The E-CDF-S is composed of four contiguous $\approx 250 \mathrm{ks}$ Chandra fields covering an $\approx 0.3 \mathrm{deg}^{2}$ region, which flanks the $\approx 1$ Ms CDF-S. A large fraction of the E-CDF-S $(\approx 80 \%)$ has been observed with HST in two ACS filters, $V_{606}$ and $z_{850}$, through the Galaxy Evolution from Morphology and SEDs (GEMS; Rix et al. 2004) survey. The E-CDF-S Chandra observations can detect $z=0.1$ off-nuclear sources with projected physical offsets of $\gtrsim 2 \mathrm{kpc}$ and $0.5-2.0 \mathrm{keV}$ luminosities of $\gtrsim 3 \times 10^{39} \mathrm{erg} \mathrm{s}^{-1}$ in the most sensitive regions. Furthermore, $z=0.1$ sources with physical offsets of $\gtrsim 3 \mathrm{kpc}$ and $0.5-2.0 \mathrm{keV}$ luminosities $\gtrsim 3 \times 10^{40} \mathrm{erg} \mathrm{s}^{-1}$ can be detected over the entire $\approx 0.3 \mathrm{deg}^{2}$ E-CDF-S field.

The Galactic column densities are $\approx 1.3 \times 10^{20} \mathrm{~cm}^{-2}$ for the CDF-N (Lockman 2003) and $\approx 8.8 \times 10^{19} \mathrm{~cm}^{-2}$ for the CDF-S and E-CDF-S (Stark et al. 1992). All of the X-ray 
fluxes and luminosities quoted throughout this paper have been corrected for Galactic absorption using these column densities. Often we quote Poisson errors with values indicating $1 \sigma$ significance levels; these are computed following Gehrels (1986). $H_{0}=70 \mathrm{~km} \mathrm{~s}^{-1} \mathrm{Mpc}^{-1}$, $\Omega_{\mathrm{m}}=0.3$, and $\Omega_{\Lambda}=0.7$ are adopted throughout this paper (Spergel et al. 2003), and the coordinates are J2000.0.

\section{Off-Nuclear Source Sample Construction}

\subsection{Sample Selection}

As discussed above, the combination of the high spatial resolution of HST imaging and sensitive X-ray observations with Chandra is effective in detecting and classifying offnuclear X-ray sources out to $z \approx 0.3$. The $\approx 2 \mathrm{Ms}$ CDF-N, $\approx 1 \mathrm{Ms}$ CDF-S, and $\approx 250 \mathrm{ks}$ E-CDF-S (hereafter CDFs) are currently the deepest extragalactic X-ray surveys conducted with Chandra (see, e.g., Brandt \& Hasinger 2005 for a review), and all of these surveys have good HST coverage. These observations reach $0.5-2.0 \mathrm{keV}$ sensitivity limits ranging from $1.8 \times 10^{-17} \mathrm{erg} \mathrm{cm} \mathrm{cm}^{-2} \mathrm{~s}^{-1}$ in the CDF-N to $1.1 \times 10^{-16} \mathrm{erg} \mathrm{cm}^{-2} \mathrm{~s}^{-1}$ in the E-CDF-S. However, as demonstrated in $\S 3.4 .2$ of Alexander et al. (2003) and $\S 3.3 .2$ of Lehmer et al. (2005a), legitimate lower-significance X-ray sources below these quoted sensitivity limits can be identified by matching such sources to associated optically bright galaxies. We therefore searched for off-nuclear sources using both the published main Chandra catalogs of Alexander et al. (2003; CDF-N and CDF-S) and Lehmer et al. (2005a; E-CDF-S) as well as additional lower-significance X-ray sources detected in these fields by running the CIAO source-searching algorithm wavdetect (Freeman et al. 2002) at a false-positive probability threshold of $1 \times 10^{-5}$. A total of 933, 689, and 1085 X-ray sources in the CDF-N, CDF-S, and E-CDF-S, respectively, were thus used in our searching; these numbers do not take into account the overlapping regions of the CDF-S and E-CDF-S.

We searched for and classified off-nuclear X-ray source candidates in the CDFs using the following criteria:

1. An X-ray source is considered to be an off-nuclear candidate if its position is offset from the optical nucleus of an optically-bright galaxy (ACS magnitudes of $V_{606}<21$; see below for a description of how this limit was selected) by $\geq 1.5 \times$ the radius of the Chandra positional error circle (see equations (2) and (3) of Alexander et al. (2003) and Lehmer et al. (2005a), respectively) but is still observed to lie within the optical extent of the candidate host galaxy. The radius of the positional error circle (80-90\% confidence) for Chandra sources detected in the CDFs ranges from $\approx 0$ "'.3-1".5 and is de- 
pendent on the Chandra point-spread function (PSF) size and the number of observed counts. Our required minimum offset of $1.5 \times$ the radius of the Chandra positional error circle was chosen empirically based on histograms showing the number of sources with X-ray-to-optical flux ratios similar to those of active galactic nuclei (AGNs) (i.e., sources with $\left.\log \left[f_{\mathrm{X}} / f_{\mathrm{V}}\right]>-0.5\right)$ versus their X-ray-to-optical positional offsets; we found that $\approx 98 \%$ of $\mathrm{AGN}$-like sources with $V_{606}<24$ were matched to within $1.5 \times$ the radius of the Chandra positional error circle. For illustrative purposes, we provide Figure 1, which shows the logarithm of the X-ray-to-optical flux ratio $\left(\log \left[f_{\mathrm{X}} / f_{\mathrm{V}}\right]\right)$ versus positional offset in units of the Chandra positional error for $0.5-2.0 \mathrm{keV}$ detected sources with optical counterparts having $V_{606}<24$. On the basis of our analysis, the number of $V_{606}<21$ nuclear sources in our sample expected to have offsets larger than $1.5 \times$ the radius of the Chandra positional error circle is calculated to be $\approx 0.91_{-0.75}^{+2.11}$.

2. We required that candidate host galaxies have redshifts in the range of $0<z \leq 0.3$. We used spectroscopic redshifts for $\approx 98 \%$ of the field galaxies in the CDF-N (e.g., Barger et al. 2003; Wirth et al. 2004) and $\approx 6 \%$ of the field galaxies in the CDF-S and E-CDF-S (e.g., Szokoly et al. 2004). For the remaining field galaxies in the CDF-N $(\approx 2 \%)$ and CDF-S and E-CDF-S $(\approx 94 \%)$, we used high-quality photometric redshifts from GOODS (Mobasher et al. 2004) and COMBO-17 (Wolf et al. 2004), respectively. We note that in the CDF-S and E-CDF-S, where the majority of the field galaxies have only photometric redshifts via the 17-filter photometry from COMBO-17, the redshift uncertainties are small (i.e., $\delta_{z} /(1+z) \approx 0.01$ ). The selected redshift range was chosen empirically by considering the observed number of off-nuclear sources detected as a function of redshift. Furthermore, for redshifts larger than 0.3, the projected linear distance corresponding to the typical Chandra positional error ( $\gtrsim 7 \mathrm{kpc}$ ) becomes unreasonably large for detecting many off-nuclear sources.

3. In an effort to minimize confusion with unrelated background X-ray sources, we further required that each off-nuclear source candidate is detected in either the $0.5-2.0 \mathrm{keV}$ or 0.5-8.0 keV bands and has a $0.5-2.0 \mathrm{keV}$ luminosity $\leq 10^{41} \mathrm{erg} \mathrm{s}^{-1}$. These restrictions are based on the observed spectral and luminosity properties of local IXOs. X-ray luminosities were computed using the equation

$$
L_{\mathrm{X}}=4 \pi d_{L}^{2} f_{\mathrm{X}}(1+z)^{\Gamma-2}
$$

where $d_{L}$ is the luminosity distance, $f_{\mathrm{X}}$ is the $\mathrm{X}$-ray flux of each source, and $\Gamma$ is the X-ray photon index assuming a power-law Spectral Energy Distribution (SED). We adopt a photon index of $\Gamma=1.8$ throughout this paper (see $\S 2.2$ for justification). 
The sky density of optically-bright galaxies is relatively low, and thus the probability of finding an unrelated background X-ray source within the optical extent of these galaxies is also low. In order to estimate the total number of background sources that may be contaminating our sample and to determine the optimal optical-magnitude limit down to which we search for off-nuclear sources, we followed a similar methodology to that outlined in $\S 2$ of PC04. We first chose a tentative optical magnitude limit down to which we search for off-nuclear sources. For each candidate host galaxy, we then visually estimated the galactic area within which we would consider an X-ray source to be off-nuclear. This area was approximated by defining an ellipse with a semimajor and semiminor axis and position angle for each galaxy with a circle of radius $1.5 \times$ the radius of the Chandra positional error circle removed. Next, we calculated the average $0.5-2.0 \mathrm{keV}$ sensitivity limit over the relevant area of each galaxy using empirically calculated sensitivity maps that were calibrated appropriately for sources detected by wavdetect using a false-positive probability threshold of $1 \times 10^{-5}$. We chose to use the $0.5-2.0 \mathrm{keV}$ band because of its low background and correspondingly high sensitivity. The number of expected background $0.5-2.0 \mathrm{keV}$ sources per galaxy was estimated using the galactic area, galaxy sensitivity limit, and best-fit numbercounts relation presented in $\S 4$ of Bauer et al. (2004). Finally, the total number of expected contaminating background sources was computed by summing the contributions from each galaxy. We found that when restricting our search to galaxies with $V_{606}<21$, the number of expected contaminating background sources in the entire survey is reasonably low $(\approx 2.22)$, so we chose $V_{606}=21$ as our optical magnitude limit. For galaxies brighter than this limit, the median (mean) number of expected background X-ray sources was $\approx 0.0029$ (0.0064) galaxy $^{-1}$. We appropriately correct for contaminating background sources in our analyses below.

\subsection{X-ray and Optical Properties of Off-Nuclear Sources and Host Galaxies}

Using the criteria presented above, we identified a total of 24 off-nuclear source candidates (see Figure 2); nine of these were previously detected by H04. The additional source presented in H04 that is not included here (CXOCDFS J033220.35-274555.3) was within $1.5 \times$ the Chandra positional error and was only detected in the $0.5-1.0 \mathrm{keV}$ band. All of the off-nuclear source candidates are coincident with late-type galaxies (i.e., spirals and irregulars); this is generally consistent with studies of IXOs in the local universe (see $\S 1$ ). We also note that two of the off-nuclear sources (CXOHDFN J123701.99+621122.1 and CXOHDFDN J123706.12+621711.9) are located in galaxies having discernable bars of

optical emission. The X-ray properties of the off-nuclear sources are summarized in Table 1 and additional properties, including those of the host-galaxies, are summarized in 
Table 2; median properties of are listed in the last rows of these Tables. The median offnuclear source is offset by $\approx 2.1 \times$ the Chandra positional error, and only two sources (CXOCDFS J033230.01-274404.0 and CXOECDFS J033322.97-273430.7) have offsets near our required minimum offset of $1.5 \times$ the Chandra positional error (see Figure 1). CXOCDFS J033230.01-274404.0 was detected and classified as being off-nuclear in both the CDF-S and E-CDF-S data sets independently, lending additional support to its off-nuclear classification.

The off-nuclear X-ray sources span a $0.5-2.0 \mathrm{keV}$ luminosity range of $8 \times 10^{38} \mathrm{erg} \mathrm{s}^{-1}$ to $6 \times 10^{40} \mathrm{erg} \mathrm{s}^{-1}$ and have a median luminosity of $\approx 8 \times 10^{39} \mathrm{erg} \mathrm{s}^{-1}$. The host galaxies have a median redshift of $z \approx 0.14$, which corresponds to a lookback time of $\approx 1.8 \mathrm{Gyr}$, and a median $V_{606}$ magnitude of 19.1. In Figure 3a, we show the X-ray luminosities of these 24 off-nuclear sources as a function of redshift. The fifteen new objects (filled symbols) presented here significantly improve the source statistics at $L_{0.5-2.0 \mathrm{keV}} \gtrsim 10^{39.5} \mathrm{erg} \mathrm{s}^{-1}$ and $z=0.15-0.3$; many of these sources are from the relatively wide solid-angle E-CDF-S survey. All sources with $0.5-2.0 \mathrm{keV}$ upper limits are detected in the $0.5-8.0 \mathrm{keV}$ band.

We constrained the average X-ray spectral shape of our off-nuclear sources by stacking the $0.5-2.0 \mathrm{keV}$ and $2-8 \mathrm{keV}$ source counts and exposures. We then computed a vignettingcorrected band ratio by taking the ratio $\Phi_{2-8 \mathrm{keV}} / \Phi_{0.5-2.0 \mathrm{keV}}$, where $\Phi$ is defined to be the $\mathrm{X}$-ray count rate in units of counts $\mathrm{s}^{-1}$. The band ratio was converted into a mean effective photon index $\Gamma_{\text {eff }}$ using the Chandra X-ray Center's Portable, Interactive, Multi-Mission Simulator (PIMMS). For the 24 off-nuclear X-ray sources, the mean effective photon index is $\Gamma_{\text {eff }}=1.83_{-0.17}^{+0.21}$, a value consistent with that for IXOs observed in the local universe (e.g., Liu \& Mirabel 2005 and references therein). We therefore adopted $\Gamma=1.8$ when computing $\mathrm{X}$-ray luminosities for our off-nuclear sources using equation (1).

Since all of our off-nuclear sources are coincident with late-type galaxies (see Figure 2), we chose to restrict further comparative analyses to galaxies with late-type morphologies. We selected galaxies in the ACS-covered regions of the CDFs and required $V_{606}<21$ in the redshift range $0<z \leq 0.3$. At the quoted optical-magnitude limit, roughly half of the objects detected are Galactic stars; these were characterized visually as point-like sources with diffraction spikes and removed from our list of candidate field galaxies. In total, 385 objects were selected as being non-stellar extragalactic sources with $V_{606}<21$ and $0<z \leq 0.3$. We visually classified each galaxy as being either an elliptical (48 galaxies) or a spiral or irregular (337 galaxies). Figure $3 \mathrm{~b}$ shows the estimated rest-frame $6000 \AA$ luminosities $\left(\nu L_{\nu}[6000 \AA]\right)$ for all of the 337 spiral and irregular galaxies in our sample (small filled circles). Galaxies hosting off-nuclear sources are plotted with larger open and filled symbols (for H04 and new sources, respectively); most of these occupy the high-luminosity end of the distribution. Optical luminosities were estimated using the $V_{606}$ magnitude and applying a $K$-correction 
assuming an Scd spiral-galaxy optical SED (Coleman et al. 1980); this SED was chosen since it is commonly observed for star-forming galaxies. We chose to use the $V_{606}$ band to compute the $6000 \AA$ luminosities because (1) it is availabile over all of the CDFs and (2) the $6000 \AA$ continuum traces the emission from relatively old stellar populations. The $K$-corrections for the galaxies are small $(\lesssim 0.4 \mathrm{mag})$ and have little dependence on galaxy SED choice. The median $6000 \AA$ luminosities $\left(6000 \AA\right.$ absolute magnitudes, $\left.M_{606}\right)$ of the intermediate-redshift field galaxies and the host galaxies of off-nuclear X-ray sources are $\nu L_{\nu}(6000 \AA) \approx 1.9 \times 10^{43} \mathrm{erg} \mathrm{s}^{-1}(-19.8)$ and $\approx 2.7 \times 10^{43} \mathrm{erg} \mathrm{s}^{-1}(-20.2)$, respectively.

Ten of our $24\left(41_{-13}^{+18} \%\right)$ off-nuclear X-ray sources appear to be coincident with optical knots of emission (see column 8 of Table 2 and the images in Figure 2). In the local universe, IXOs often appear to be located within or near star-forming regions, ranging from small ( $<100$ pc diameter) diffuse $\mathrm{H} \alpha$ emission complexes to giant ( $\gtrsim 500$ pc diameter) H II regions (e.g., Pakull \& Mirioni 2002; Liu \& Bregman 2005; Ramsey et al. 2006). The optical knots in our sample have apparent diameters of $\approx 500-1000$ pc and optical luminosities of $\nu L_{\nu}(6000 \AA) \approx 10^{40-41} \mathrm{erg} \mathrm{s}^{-1}$. These optical properties are broadly consistent with those of giant H II regions in the local universe (e.g., Kennicutt 1984), suggesting these off-nuclear sources trace distant star-formation regions. In the GOODS fields, high-resolution ACS imaging is available over four optical bands $\left(B_{435}, V_{606}, i_{775}\right.$, and $\left.z_{850}\right)$; for the eight offnuclear sources with optical knots located in these ACS regions, we analyzed the colors in the immediate vicinity of the knots. Figure 4 shows the relative color difference between the optical knots and their host galaxies. These relative colors are defined as follows:

$$
\begin{aligned}
\Delta\left(B_{435}-V_{606}\right) & \equiv\left(B_{435}-V_{606}\right)_{\text {knot }}-\left(B_{435}-V_{606}\right)_{\text {galaxy }} \\
\Delta\left(V_{606}-i_{775}\right) & \equiv\left(V_{606}-i_{775}\right)_{\text {knot }}-\left(V_{606}-i_{775}\right)_{\text {galaxy }}
\end{aligned}
$$

We find that the optical knots are relatively blue compared to their host galaxies (i.e., mean values of $\Delta\left(B_{435}-V_{606}\right)=-0.22 \pm 0.14$ and $\Delta\left(V_{606}-i_{775}\right)=-0.13 \pm 0.18$ are observed $)$. We stacked the $0.5-2.0 \mathrm{keV}$ and 2-8 keV counts from our off-nuclear sources with and without optical knots to see if we could distinguish between the mean effective photon indices $\left(\Gamma_{\text {eff }}\right.$; see text above) of these two populations. We found that the average spectral shapes of these two populations are statistically consistent (i.e., $\Gamma_{\text {eff }}=1.90_{-0.21}^{+0.26}$ and $1.70_{-0.28}^{+0.41}$, for sources with and without optical knots, respectively).

We also investigated the optical colors of the host galaxies to see if the off-nuclear sources are found in more actively star-forming galaxies. We utilized $B$ - and $V$-band magnitudes from ground-based observations of the CDF-N with the Subaru $8.2 \mathrm{~m}$ telescope (Capak et al. 2004), and of the CDF-S and E-CDF-S with the Wide Field Imager of the MPG/ESO 
telescope at La Silla (see $\S 2$ of Giavalisco et al. 2004); small corrections for the differing bandpasses were applied to the CDF-S and E-CDF-S magnitudes to match those in the CDF-N. Optical emission lines produced by star-forming galaxies can influence the observed broad-band fluxes. At the median redshift of our off-nuclear source sample $(z=0.14)$, the strong nebular emission line due to $[\mathrm{O}$ II] $\lambda 3727$ is located in the $B$-band and the redder emission lines from the [O III] $\lambda \lambda 4959,5007$ doublet and $\mathrm{H} \beta \lambda 4861$ are located in the $V$-band. In Figure 5, we show the $B-V$ colors vs. $V_{606}$-band magnitudes for our 337 spiral and irregular galaxies in the CDFs. Field galaxies hosting off-nuclear sources have been outlined with open squares and circles, which distinguish between sources with and without optical knots, respectively. The mean $B-V$ optical colors have been plotted for the galaxies (solid line), galaxies hosting off-nuclear sources (dot-dashed line), and galaxies

hosting off-nuclear sources with (dashed line) and without (dotted line) optical knots. The typical galaxy hosting an off-nuclear X-ray source shows somewhat bluer optical colors than typical galaxies in the field. We utilized the two-sample Kolmogorov-Smirnov (K-S) test on the unbinned $B-V$ samples of field galaxies and galaxies hosting off-nuclear sources, and repeated the test to compare the $B-V$ sample of field galaxies with off-nuclear sources with and without optical-knot counterparts. We found that, at the $\approx 89 \%$ confidence level (i.e., K-S probability $\approx 0.11$ ), the $B-V$ colors of galaxies hosting off-nuclear sources are statistically different from typical field galaxies in the CDFs. Furthermore, the colors of galaxies hosting off-nuclear sources with optical knots are statistically different from those of typical field galaxies at the $\approx 96 \%$ confidence level, but galaxies hosting off-nuclear sources without optical knots have colors statistically consistent with those of typical field galaxies. These tests suggest that off-nuclear sources are indeed preferentially located in galaxies undergoing intense star formation; the optical knots directly trace star-forming complexes in such galaxies. For comparison, we plotted the distribution of Johnson $U-B$ colors versus $V$-band magnitude for RC3 galaxies (inset to Figure 5) and highlighted six local galaxies (NGC 1566, NGC 1672, NGC 3623, NGC 4088, NGC 4303, and NGC 4490) observed to host IXOs coincident with distinct, luminous optical knots from Liu \& Bregman (2005). These galaxies appear to be notably bluer than typical RC3 galaxies, and using the K-S test, we find that the colors of these galaxy samples are statistically different at the $\approx 96 \%$ confidence level. Locally, the $U$ - and $B$-band magnitudes sample the strong emission features expected in the $B$ - and $V$-bands, respectively, at $z=0.14$.

\section{Analysis and Results}

A primary goal of this investigation is to determine whether the "true" luminositydependent fraction of galaxies containing off-nuclear X-ray sources $\left(f_{\mathrm{T}}\right)$ evolves with cos- 
mic time (see $\S 1$ ). Below, we describe our procedure for computing this fraction for our intermediate-redshift galaxy sample and a matched comparison sample of local galaxies from PC04. We assess observational constraints on the X-ray luminosity detection limit and angular resolution for both our sample and that of PC04. To this end, we first describe the computation of the luminosity-dependent "observed" fraction of galaxies hosting off-nuclear sources $\left(f_{\mathrm{O}}\right)$; this takes into account the spatially varying sensitivity of the Chandra observations. We then estimate the true fraction $\left(f_{\mathrm{T}}\right)$ by calculating correction factors to $f_{\mathrm{O}}$, which account for (1) the number of off-nuclear sources we expect to miss due to angular-resolution limitations (i.e., the number of off-nuclear sources with offsets smaller than the resolution limit) and (2) the multiplicity of these sources within host galaxies (i.e., the expected number of off-nuclear sources within a galaxy having at least one off-nuclear source).

\subsection{The Observed Fraction $\left(f_{\mathrm{O}}\right)$}

To compute the observed fraction of intermediate-redshift galaxies hosting off-nuclear $\mathrm{X}$-ray sources, we followed a procedure similar to that outlined in $\S 2$ of PC04. For each of our 337 spiral and irregular galaxies, we used the $0.5-2.0 \mathrm{keV}$ sensitivity maps described in $\S 2.1$ and corresponding redshift information to compute an X-ray luminosity limit above which we could detect an off-nuclear source. All luminosities were calculated using equation (1), adopting $\Gamma=1.8$. In Figure 6a, we show the number of galaxies with $\mathrm{X}$-ray coverage sensitive enough to detect an off-nuclear source of a given $0.5-2.0 \mathrm{keV}$ luminosity, $L_{\mathrm{X}}$. For example, there are 20 galaxies with X-ray coverage sensitive enough to detect an off-nuclear source with $L_{\mathrm{X}} \approx 10^{38.9} \mathrm{erg} \mathrm{s}^{-1}$ (i.e., the leftmost bin of Figure 6a), and there are 337 galaxies with coverage sensitive enough to detect an off-nuclear source with $L_{\mathrm{X}} \approx 10^{41} \mathrm{erg} \mathrm{s}^{-1}$ (i.e., the rightmost bin of Figure $6 \mathrm{a}$ ). Figure $6 \mathrm{~b}$ shows the number of galaxies in each $L_{\mathrm{X}}$ bin of Figure 6a observed to host an off-nuclear source of $L_{\mathrm{X}}$ or greater. In Figure $6 \mathrm{~b}$, the number of galaxies included in a given $L_{\mathrm{X}}$ bin versus its neighboring lower-luminosity $L_{\mathrm{X}}$ bin is affected by both the addition of galaxies hosting off-nuclear sources with less sensitive X-ray coverage and the subtraction of galaxies hosting off-nuclear sources that fall below $L_{\mathrm{X}}$. In order to aid in the understanding of this progression, we have created Figures $6 \mathrm{c}$ and $6 \mathrm{~d}$, which show the number of added and subtracted galaxies (in ascending order from $L_{\mathrm{X}} \approx 10^{38.9} \mathrm{erg} \mathrm{s}^{-1}$ to $L_{\mathrm{X}} \approx 10^{41} \mathrm{erg} \mathrm{s}^{-1}$ ), respectively. For example, six galaxies in the first $L_{\mathrm{X}}$ bin of Figure $6 \mathrm{a}$ that host off-nuclear sources with $L_{\mathrm{X}} \approx 10^{38.9} \mathrm{erg} \mathrm{s}^{-1}$ are added to the first $L_{\mathrm{X}} \approx 10^{38.9}$ $\mathrm{erg} \mathrm{s}^{-1}$ bin of Figure $6 \mathrm{~b}$. In the next higher $L_{\mathrm{X}}$ bin (i.e., $L_{\mathrm{X}} \approx 10^{39.3} \mathrm{erg} \mathrm{s}^{-1}$ ), two new

galaxies hosting off-nuclear sources with $L_{\mathrm{X}} \gtrsim 10^{39.3} \mathrm{erg} \mathrm{s}^{-1}$ are added to the appropriate $L_{\mathrm{X}}$ bin of Figure $6 \mathrm{~b}$, and two galaxies with off-nuclear source luminosities $<10^{39.3} \mathrm{erg} \mathrm{s}^{-1}$ are subtracted from the same bin. Thus the total number of galaxies with off-nuclear sources 
of $L_{\mathrm{X}} \geq 10^{39.3} \mathrm{erg} \mathrm{s}^{-1}$ remains at six.

In order to calculate the observed fraction of intermediate-redshift galaxies hosting offnuclear sources $\left(f_{\mathrm{O}}\right)$, we divide the histogram entries of Figure 6b, with the estimated number of background sources subtracted (see $\S 2.1$ ), by those of Figure 6a; the observed fraction is presented in Figure 7 as a dashed line with shaded $1 \sigma$ error envelope (computed following Gehrels 1986). The dashed line terminates for $L_{\mathrm{X}} \gtrsim 10^{40.7} \mathrm{erg} \mathrm{s}^{-1}$ due to the fact that there are no off-nuclear sources in our sample with luminosities exceeding $10^{40.7} \mathrm{erg} \mathrm{s}^{-1}$; the shaded region therefore represents the $3 \sigma$ upper limit to $f_{\mathrm{O}}$ for $L_{\mathrm{X}} \gtrsim 10^{40.7} \mathrm{erg} \mathrm{s}^{-1}$. To make comparisons between the observed fraction of our intermediate-redshift sample and that of the local sample of PC04, we matched the optical-luminosity distribution of the PC04 spiral and irregular galaxies (i.e., Hubble type $\geq 0$ ) to that of our 337 spiral and irregular galaxies. At the median redshift $(z=0.14)$ of our off-nuclear X-ray source sample, the $V_{606}<21$ requirement on the inclusion of galaxies in our analyses equates to requiring the rest-frame optical luminosity of a particular galaxy to be $\nu L_{\nu}(6000 \AA) \gtrsim 10^{42.6} \mathrm{erg} \mathrm{s}^{-1}$ $\left(M_{606} \lesssim-18.1\right.$; see Figure $\left.3 \mathrm{~b}\right)$. We created a matched PC04 subsample by applying the same optical luminosity cut to the original PC04 spiral and irregular sample. In total, 329 spiral and irregular galaxies were selected for comparison from the original PC04 sample of 766 galaxies. Figure 8 shows the $\nu L_{\nu}(6000 \AA)$ luminosity distributions of galaxies for both our sample (solid histogram) and the matched subsample of PC04 (dotted histogram). The two luminosity distributions span roughly the same $\nu L_{\nu}(6000 \AA)$ range and have similar overall shapes; a K-S test indicates that these two distributions are statistically consistent for $\nu L_{\nu}(6000 \AA) \gtrsim 10^{42.6} \mathrm{erg} \mathrm{s}^{-1}$ (i.e., K-S probability $\left.\approx 0.6\right)$. Using the matched $\mathrm{PC} 04$ subsample and the procedure described above, we recomputed the relevant observed fraction of local galaxies hosting IXOs $\left(f_{\mathrm{O}, \mathrm{PC} 04}\right)$; this is shown in Figure 7 as filled circles with $1 \sigma$ error bars and $3 \sigma$ upper limits.

\subsection{The True Fraction $\left(f_{\mathrm{T}}\right)$}

As noted above, the angular resolution of Chandra, while superb, is limited to the classification of off-nuclear sources at intermediate-redshifts that are offset by more than 1.5 $\times$ the Chandra positional error from the optical position of the galactic nucleus. Furthermore, the classification of IXOs in the PC04 sample is also limited to X-ray sources offset by $\gtrsim 10^{\prime \prime}$ (Colbert \& Ptak 2002). Therefore, the observed fractions for both our sample and the matched PC04 subsample can only be considered to be lower limits on the true fractions of galaxies hosting off-nuclear sources, and therefore direct comparison between these two samples is not meaningful. To address this problem, we have computed the relevant scal- 
ing between the observed and true fractions (for both our sample and the matched PC04 subsample) through simulations using a sample of local IXO-hosting galaxies observed with Chandra; we describe these simulations below.

We calculated the distribution of projected physical offsets of 65 IXOs from 29 Chandra-observed local $(D \lesssim 50 \mathrm{Mpc}$ ) galaxies (compiled by Liu \& Mirabel 2005; see references therein). Observations of IXOs in local galaxies with Chandra should be statistically complete for sources with offsets $\gtrsim 0.1 \mathrm{kpc}$, assuming a positional accuracy of $\approx 0 ! 5$ and a maximal galactic distance of $\approx 50 \mathrm{Mpc}$. The normalized distribution of offsets for the 65 IXOs used here is shown in Figure 9 (solid histogram). Similar distributions were calculated using subsets of these IXOs with $0.5-2.0 \mathrm{keV}$ luminosities $>10^{39-39.5} \mathrm{erg} \mathrm{s}^{-1}$; no strong luminosity dependence was observed in the shape of these curves. The overall offset distribution shown in Figure 9 indicates that IXOs are often located relatively close to their host-galaxy nucleus, which is considerably different from the scenario where IXOs are distributed throughout galaxies with constant density (dotted curve). This observed distribution is likely due to the high circumnuclear star-formation activity commonly found in normal and starburst galaxies (e.g., Kennicutt 1998) and is consistent with the IXO offset distribution noted by Swartz et al. (2004). The median physical offset for the 65 Chan$d r a$-observed local IXOs is $\approx 3 \mathrm{kpc}$ (vertical dashed line) and the expected resolution for CDF sources at the median redshift $(z=0.14)$ of our off-nuclear source sample is $\approx 2.6 \mathrm{kpc}$ (vertical dot-dashed line); to first order, this suggests that our observed off-nuclear sources only make up $\sim 50 \%$ of the physical offset distribution, and we are plausibly missing about half of the off-nuclear sources.

Next, we computed the fraction of galaxies in which an X-ray source of a given linear offset would be classified as "off-nuclear" for galaxies in both our intermediate-redshift galaxy sample and the matched PC04 subsample. The relevant fractions for each sample are shown as solid curves in the top panels of Figure 10; dotted curves show the case where there is no resolution limitation. These offset-dependent fractions are constrained by both the projected physical resolution (the observed rise in the fraction near small offsets) and the projected optical size of the galaxies (the observed decline in the fraction at larger offsets). The angular resolution was taken to be $1.5 \times$ the Chandra positional error for our galaxy sample and was fixed at $10^{\prime \prime}$ for the matched PC04 subsample. The angular size of each galaxy was assumed to be the apparent optical semimajor axis (see $\S 2.1$ ) and $0.5 \times$ the RC3 major-axis diameter $\left(D_{25}\right)$ for our sample and the matched PC04 subsample, respectively. The solid curves presented in the top panels of Figure 10 for each sample are significantly different in the small-offset regime, showing that our sample is more heavily affected by angular-resolution limitations than the matched PC04 subsample. We simulated the expected observable offset distribution of each galaxy sample by convolving the local offset distribution presented in 
Figure 9 (solid histogram) with the detectable fraction curves (top panels of Figure 10; solid curves); these are displayed as solid histograms in the bottom panels of Figure 10. For comparison, we have shown the actual observed offset distributions of off-nuclear sources in our sample and the matched PC04 subsample (dashed histograms in the bottom panels of Figure 10, respectively). By inspection, we find that the observed and simulated distributions are consistent.

In order to estimate the number of off-nuclear sources that were missed due to instrumentalresolution limitations, we simulated the expected distributions of offsets for off-nuclear sources in each galaxy sample for the case where there is no resolution limitation. This was done by convolving the local offset distribution function (solid histogram in Figure 9) with the dotted curves in the top panels of Figure 10. These resulting distribution functions, normalized by the simulated observed distribution functions (solid histograms in the bottom panels of Figure 10), are presented as dotted histograms in the bottom panels of Figure 10. These calculations suggest that $62.1 \%$ and $35.0 \%$ of the off-nuclear sources would remain unclassified as "off-nuclear" for our intermediate-redshift sample and the matched PC04 subsample, respectively.

In the simple case where we would expect $\approx 1$ off-nuclear source per galaxy, we could simply rescale the observed fractions by a constant scaling factor to obtain the true fractions; we define this scaling factor as $\alpha_{\mathrm{s}}$, which is 2.64 and 1.54 for our off-nuclear source sample and the matched PC04 subsample, respectively. However, observations of IXOs in the local universe, show that, on average, there is more than one IXO per IXO-hosting galaxy (e.g., Colbert \& Ptak 2002). Since we observe only one IXO per IXO-hosting galaxy for our intermediate-redshift sample, a simple constant scaling of the observed fraction to obtain the true fraction is not appropriate. Hereafter, we refer to the mean number of observed IXOs per IXO-hosting galaxy as the "multiplicity" factor $(m)$, which varies with IXO luminosity; using the Liu \& Mirabel (2005) sample of IXOs, we have estimated the "true" luminosity-dependent multiplicity factor $\left(m_{\mathrm{T}}\right)$. We find that $m_{\mathrm{T}}$ has a value of $\approx 2.1$ and $\approx 1.0$ IXOs per IXOhosting galaxy for $0.5-2.0 \mathrm{keV}$ luminosities of $L_{\mathrm{X}} \gtrsim 10^{38.9} \mathrm{erg} \mathrm{s}^{-1}$ and $L_{\mathrm{X}} \gtrsim 10^{40} \mathrm{erg} \mathrm{s}^{-1}$, respectively; for comparison, the corresponding observed PC04 multiplicity factor ( $\left.m_{\mathrm{O}, \mathrm{PC} 04}\right)$ has a value of $\approx 1.5$ and $\approx 1.0$ IXOs per IXO-hosting galaxy, respectively. Using the scaling factor $\alpha_{\mathrm{s}}$, the observed multiplicity factor $m_{\mathrm{O}}$, and the true multiplicity factor $m_{\mathrm{T}}$, we converted the observed fraction $f_{\mathrm{O}}$ to the true fraction $f_{\mathrm{T}}$ following

$$
f_{\mathrm{T}}=\alpha_{\mathrm{s}} \frac{m_{\mathrm{O}}}{m_{\mathrm{T}}} f_{\mathrm{O}}
$$

The luminosity-dependent scaling factors (for our intermediate-redshift sample and the 
matched PC04 subsample), $\alpha_{\mathrm{s}} m_{\mathrm{O}} / m_{\mathrm{T}}$, which scale $f_{\mathrm{O}}$ to obtain $f_{\mathrm{T}}$, are shown in Figure 11a; the resulting true fractions with $1 \sigma$ errors (computed following Gehrels 1986) are shown in Figure 11b. Fractions measured for our sample $\left(f_{\mathrm{T}, \text { int }-z}\right)$ are shown as the dashed line surrounded by the shaded error envelope, and the matched PC04 fractions $\left(f_{\mathrm{T}, \mathrm{PC} 04}\right)$ are plotted as filled black circles with $1 \sigma$ error bars and $3 \sigma$ upper limits. The dotted curve shows the fraction of our galaxies with an X-ray source of $0.5-2.0 \mathrm{keV}$ luminosity $L_{\mathrm{X}}$ or greater (including both nuclear and off-nuclear sources). This curve was obtained by matching galaxies in our sample to X-ray sources from the main and supplementary Chandra catalogs of the CDFs using a matching criterion of less than one semimajor axis length. Matched sources may include a variety of X-ray sources such as AGNs, luminous nuclear starbursts, and off-nuclear X-ray sources with both small and large offsets (i.e., offsets both less than and greater than $1.5 \times$ the radius of the Chandra positional error circle). This curve represents an upper limit to the true fraction of galaxies hosting off-nuclear sources, and we note that our calculated true fraction is below this limit. Furthermore, based on this curve, we calculate that off-nuclear sources are found in $\approx 75 \%$ and $\approx 40 \%$ of the $z \leq 0.3$ galaxies detected in the $0.5-2.0 \mathrm{keV}$ bandpass with coverage sensitive enough to detect sources of $L_{\mathrm{X}}>10^{39}$ $\mathrm{erg} \mathrm{s}^{-1}$ and $L_{\mathrm{X}}>10^{40.7} \mathrm{erg} \mathrm{s}^{-1}$, respectively. For further comparison, we have plotted the fraction of galaxies with an X-ray source of $0.5-2.0 \mathrm{keV}$ luminosity $L_{\mathrm{X}}$ or greater but with a luminosity upper bound of $10^{41.5} \mathrm{erg} \mathrm{s}^{-1}$ (dot-dashed curve); this upper limit was adopted to include mostly galaxies powered by star-forming processes. Again, our estimate of $f_{\mathrm{T}, \text { int }-z}$ appears to be reasonable in comparison to this detection fraction.

Figure 11b shows suggestively that the off-nuclear source frequency for field galaxies rises with redshift. We estimate that $\approx 31_{-16}^{+20} \%$ of intermediate-redshift spiral and irregular galaxies with $\nu L_{\nu}(6000 \AA) \gtrsim 10^{42.6} \mathrm{erg} \mathrm{s}^{-1}$ host off-nuclear sources with $L_{\mathrm{X}} \gtrsim 10^{39} \mathrm{erg} \mathrm{s}^{-1}$ versus $\approx 16_{-4}^{+5} \%$ in the local universe (errors are $1 \sigma$ ). As mentioned in $\S 1$, one may plausibly expect that the frequency of off-nuclear sources would rise as a function of redshift due to the observed global increase in star-formation density, which is measured to be $\approx 1.2-3.0$ times higher at $z \approx 0.05-0.3$ than it is in the local universe (e.g., Pérez-González et al. 2005; Schiminovich et al. 2005). Furthermore, since the number of IXOs in spiral and irregular galaxies is observed to increase linearly with star-formation rate (e.g., Swartz et al. 2004), it is reasonable to expect that the frequency of off-nuclear source incidence for field galaxies would roughly scale linearly with the star-formation density. In Figure 12 we show the ratio of off-nuclear source incidence fraction for our intermediate-redshift sample and the matched PC04 subsample (i.e., $f_{\mathrm{T}, \text { int }-z} / f_{\mathrm{T}, \mathrm{PC} 04}$ ) as filled circles with $1 \sigma$ error bars. Errors on this quantity were computed using the error propogation methodology outlined in $\S 1.7 .3$ of Lyons (1991). The fraction ratio for off-nuclear sources with $L_{\mathrm{X}} \gtrsim 10^{39} \mathrm{erg} \mathrm{s}^{-1}$ is $\approx 1.9_{-1.1}^{+1.4}$; this is elevated from unity at the $\approx 80 \%$ confidence level. The dashed horizontal line shows 
the median fraction ratio. The shaded region shows the expected ratios for the case where the off-nuclear source incidence fraction scales with star-formation density; the dotted horizontal line shows the case where there is no evolution. We note that these computed ratios appear to be broadly consistent with the expected scaling of off-nuclear source incidence with redshift due to the increased global star-formation density.

\subsection{Consistency Check}

We have performed consistency checks on the results above by degrading the resolution of the PC04 subsample to match that of our intermediate-redshift sample and doing similar analyses to those above. This was achieved by calculating the median physical resolution of our intermediate-redshift galaxy sample (i.e., the median physical offset corresponding to $1.5 \times$ the Chandra positional error) and generating a subsample of PC04 IXOs with offsets greater than this median offset. Using this subset of IXOs, we calculated the resolution-normalized observed fraction $f_{\mathrm{O}, \mathrm{PC} 04}^{\text {nom }}$. In comparison to the intermediate-redshift observed fraction $f_{\mathrm{O} \text {,int-z }}$, we find results consistent with those presented above. For exam-

ple, we find that for sources with $L_{\mathrm{X}} \gtrsim 10^{39} \mathrm{erg} \mathrm{s}^{-1}$, the resolution-normalized fraction ratio $f_{\mathrm{O}, \text { int }-z} / f_{\mathrm{O}, \mathrm{PC} 04}^{\text {norm }} \approx 2.7_{-1.3}^{+2.0}$.

\section{Summary and Future Work}

We have presented the largest sample to date of intermediate-redshift $\left(z \lesssim 0.3 ; z_{\text {median }}=0.14\right)$, off-nuclear X-ray sources hosted in optically-bright $\left(V_{606}<21\right)$ field galaxies in the Chan$d r a$ deep fields. These off-nuclear X-ray sources were found to have similar X-ray spectral shapes and optical environments to IXOs in the local universe and are exclusively found to be coincident with late-type spiral and irregular galaxies. Using this sample, we found that the fraction of spiral and irregular galaxies hosting an off-nuclear X-ray source as a function of X-ray luminosity is suggestively higher at intermediate-redshifts; for off-nuclear sources with $0.5-2.0 \mathrm{keV}$ luminosities $\gtrsim 10^{39} \mathrm{erg} \mathrm{s}^{-1}$, this fraction is measured to be $\approx 31_{-16}^{+20} \%$ for intermediate-redshift field galaxies versus $\approx 16_{-4}^{+5} \%$ for local galaxies (see Figure 11 ). In computing this fraction, we have accounted for the facts that (1) the X-ray sensitivity limit varies spatially over these fields and (2) the angular resolution of Chandra limits the classification of off-nuclear sources.

Although the angular-resolution limitations of Chandra would remain, the current situation could still be improved by future Chandra observations over these fields. We note 
that only $\approx 20$ of the spiral and irregular galaxies $(\approx 6 \%)$ in our sample have $\mathrm{X}$-ray coverage sensitive enough to detect off-nuclear sources of $0.5-2.0 \mathrm{keV}$ luminosity $L_{\mathrm{X}} \approx 10^{39} \mathrm{erg} \mathrm{s}^{-1}$ (see Figure $6 \mathrm{a}$ ), a regime where $\approx 31 \%$ of the galaxies are expected to host off-nuclear X-ray sources; these sources are dispersed over all of the CDFs. Deeper Chandra observations over the CDFs would not only improve the source statistics in this regime but would also improve the positional accuracies of brighter sources too. In the $\approx 2 \mathrm{Ms}$ CDF-N, sources near the aim point that have sufficient photon counts have positional accuracies of $\approx 00^{\prime \prime} 3$. At this resolution, field galaxies at $z=0.1$ and $z=0.3$ with off-nuclear sources offset by $\gtrsim 0.8 \mathrm{kpc}$ and $\gtrsim 2.0 \mathrm{kpc}$, respectively, could be identified, and more stringent constraints could be placed on the statistical properties of these sources. Such observations covering the presently-classified off-nuclear sources would improve our knowledge of their spectral and variability properties. Furthermore, we note that other programs that utilize deep Chandra exposures in combination with HST observations (e.g., the Extended Groth Strip; Nandra et al. 2005) could build upon the present intermediate-redshift, off-nuclear source sample and improve the statistical constraints on their frequency in field galaxies.

Presently, Chandra is the only observatory capable of classifying and characterizing intermediate-redshift, off-nuclear X-ray sources. X-ray missions of the relatively near future (e.g., Constellation-X and $X E U S)^{1}$ will be capable of placing tighter constraints on the spectral and temporal properties of these sources. However, our understanding of the statistical properties of this population and its evolution with cosmic time can only be substantially improved by future X-ray missions with subarcsecond imaging capabilities such as Generation- $X,{ }^{2}$ which is planned to have imaging ( $\approx 0^{\prime \prime} 11$ resolution) and sensitivity capabilities which greatly supersede those already available through Chandra. At $z \approx 2$, an off-nuclear source with an X-ray luminosity of $\approx 10^{39} \mathrm{erg} \mathrm{s}^{-1}$ and a physical offset of $\gtrsim 0.8 \mathrm{kpc}$ could be detected and classified in a moderate-length Generation- $X$ exposure. Somewhat more luminous off-nuclear sources $\left(L_{\mathrm{X}} \gtrsim 10^{39.5} \mathrm{erg} \mathrm{s}^{-1}\right)$ with offsets as small as $\approx 0.6 \mathrm{kpc}$ could be isolated using Generation- $X$ at $z \approx 4$.

Our results suggest that the majority of the X-ray activity from normal and starburst galaxies can be explained by the presence of luminous $\left(L_{\mathrm{X}} \gtrsim 10^{39} \mathrm{erg} \mathrm{s}^{-1}\right)$ off-nuclear X-ray sources (see, e.g., Figure 11b); this is consistent with studies of X-ray point-source populations in local galaxies (e.g., Colbert et al. 2004). As discussed in $\S 1$, the global star formation rate increases with redshift, and the mean X-ray luminosity for field galaxies also

\footnotetext{
${ }^{1}$ For further information regarding Constellation-X and XEUS, visit http://constellation.gsfc.nasa.gov/ and http://www.rssd.esa.int/XEUS/, respectively.

${ }^{2}$ For further information regarding Generation- $X$, visit http://genx.cfa.harvard.edu.
} 
rises as a function of redshift. If this increase in the X-ray emission is indeed mainly due to the presence of many luminous off-nuclear sources associated with star formation, then we would expect that for distant, energetic star-forming galaxies such as Lyman break galaxies (LBGs), there may be significant crowding of luminous off-nuclear sources. The mean $0.5-2.0 \mathrm{keV}$ luminosity for LBGs at $z \approx 3$ is $\approx 10^{41} \mathrm{erg} \mathrm{s}^{-1}$ (e.g., Lehmer et al. 2005b). If we assume that the majority of this emission is coming from luminous off-nuclear sources, then we may expect that the average LBG would have $\approx 5-10$ off-nuclear sources each with $L_{\mathrm{X}} \approx 10^{39.9} \mathrm{erg} \mathrm{s}^{-1}$. When adopting an angular radius of $\approx 0^{\prime \prime} 3$ for a typical $z \approx 3 \mathrm{LBG}$ (e.g., Ferguson et al. 2004), we find that the corresponding off-nuclear source density and mean angular separation would be $\approx 20-40 \operatorname{arcsec}^{-2}$ and $\approx 0^{\prime \prime} 16-0^{\prime \prime} \cdot 22$, respectively. Future missions such as Generation- $X$ would suffer from non-negligible source confusion from the multiple off-nuclear sources within these distant LBGs.

We thank Andrew Ptak for sharing data and Mike Eracleous, Caryl Gronwall, and Ohad Shemmer for useful suggestions and discussions. We thank the anonymous referee for detailed comments and suggestions, which have improved this manuscript. We gratefully acknowledge the financial support of NSF CAREER award AST-9983783 (B.D.L., W.N.B.), Chandra Xray Center grant G04-5157A (B.D.L., W.N.B., A.T.S.), the Royal Society (D.M.A.), the Chandra Fellowship program (F.E.B.), and NSF grant AST 03-07582 (D.P.S.). 


\section{REFERENCES}

Alexander, D. M., et al. 2003, AJ, 126, 539

Barger, A. J., et al. 2003, AJ, 126, 632

Bauer, F.E., Alexander, D.M., Brandt, W.N., Schneider, D.P., Treister, E., Hornschemeier, A.E., \& Garmire, G.P. 2004, AJ, 128, 2048

Brandt, W.N., \& Hasinger, G. 2005, ARA\&A, 43, 827

Capak, P., et al. 2004, AJ, 127, 180

Colbert, E. J. M., \& Ptak, A. F. 2002, ApJS, 143, 25

Colbert, E. J. M., Heckman, T. M., Ptak, A. F., Strickland, D. K., \& Weaver, K. A. 2004, ApJ, 602, 231

Colbert, E. J. M., \& Miller, M. C. 2004 (astro-ph/0402677)

Coleman, G. D., Wu, C.-C., \& Weedman, D. W. 1980, ApJS, 43, 393

de Vaucouleurs, G., de Vaucouleurs, A., Corwin, H. G., Buta, R. J., Paturel, G., \& Fouque, P. 1991, Volume 1-3, XII, 2069 pp. 7 figs.. Springer-Verlag Berlin Heidelberg New York

Freeman, P.E., Kashyap, V., Rosner, R., \& Lamb, D.Q. 2002, ApJS, 138, 185

Ferguson, H. C., et al. 2004, ApJ, 600, L107

Gehrels, N. 1986, ApJ, 303, 336

Ghosh, P., White, N. E. 2001, ApJ, 559, 97

Giacconi, R., et al. 2002, ApJS, 139, 369

Giavalisco, M., et al. 2004, ApJ, 600, L93

Gilfanov, M., Grimm, H.-J., \& Sunyaev, R. 2004, Nuclear Physics B Proceedings Supplements, 132,369

Hornschemeier, A. E., Brandt, W. N., Alexander, D. M., Bauer, F. E., Garmire, G. P., Schneider, D. P., Bautz, M. W., \& Chartas, G. 2002, ApJ, 568, 82

Hornschemeier, A. E., et al. 2004, ApJ, 600, L147 (H04) 
Irwin, J. A., Bregman, J. N., \& Athey, A. E. 2004, ApJ, 601, L143

Kennicutt, R. C. 1984, ApJ, 287, 116

Kennicutt, R. C. 1998, ARA\&A, 36, 189

Kilgard, R. E., Kaaret, P., Krauss, M. I., Prestwich, A. H., Raley, M. T., \& Zezas, A. 2002, ApJ, 573, 138

King, A. R., Davies, M. B., Ward, M. J., Fabbiano, G., \& Elvis, M. 2001, ApJ, 552, L109

Lehmer, B. D., et al. 2005a, ApJS, 161, 21

Lehmer, B. D., et al. 2005b, AJ, 129, 1

Liu, J.-F., \& Bregman, J. N. 2005, ApJS, 157, 59

Liu, Q. Z., \& Mirabel, I. F. 2005, A\&A, 429, 1125

Lockman, F. 2003, Soft X-ray Emission from Clusters of Galaxies and Related Phonomena, ed. R. Lieu (astro-ph/0311386)

Loewenstein, M., Angelini, L., \& Mushotzky, R. F. 2005, Chinese Journal of Astronony and Astrophysics, 5, 159

Lyons, L. 1991, Data Analysis for Physical Science Students. Cambridge University Press, Cambridge

Madau, P., Pozzetti, L., \& Dickinson, M. 1998, ApJ, 498, 106

Miller, J. M., Fabian, A. C., \& Miller, M. C. 2004, ApJ, 614, L117

Mobasher, B., et al. 2004, ApJ, 600, L167

Nandra, K., et al. 2005, MNRAS, 356, 568

Pakull, M. W., \& Mirioni, L. 2002, in New Visions of the X-Ray Universe in the XMMNewton and Chandra Era, ed. F. Jansen et al. (astro-ph/0202488)

Pérez-González, P. G., et al. 2005, ApJ, 630, 82

Ptak, A., \& Colbert, E. 2004, ApJ, 606, 291 (PC04)

Ramsey, C. J., Williams, R. M., Gruendl, R. A., Chen, C.-H. R., Chu, Y.-H., \& Wang, Q. D. 2006, ApJ, in-press, (astro-ph/0511540) 
Rix, H., et al. 2004, ApJS, 152, 163

Schiminovich, D., et al. 2005, ApJ, 619, L47

Spergel, D. N., et al. 2003, ApJS, 148, 175

Stark, A. A., Gammie, C. F., Wilson, R. W., Bally, J., Linke, R. A., Heiles, C., \& Hurwitz, M. 1992, ApJS, 79, 77

Swartz, D. A., Ghosh, K. K., Tennant, A. F., \& Wu, K. 2004, ApJS, 154, 519

Szokoly, G. P., et al. 2004, ApJS, 155, 271

Wirth, G. D., et al. 2004, AJ, 127, 3121

Wolf, C., et al. 2004, A\&A, 421, 913 
Table 1. Off-Nuclear Sources: X-ray Properties

\begin{tabular}{|c|c|c|c|c|c|c|c|c|}
\hline $\begin{array}{c}\text { Source Name } \\
\text { (1) }\end{array}$ & $\begin{array}{l}\text { X-ray } \\
0.5-2.0 \mathrm{keV} \\
(2)\end{array}$ & $\begin{array}{l}\text { Counts } \\
0.5-8.0 \mathrm{keV} \\
\text { (3) }\end{array}$ & $\begin{array}{l}\Gamma \\
(4)\end{array}$ & $\begin{array}{c}f_{0.5-2.0 \mathrm{keV}} \\
\left(10^{-16}[\mathrm{cgs}]\right) \\
(5)\end{array}$ & $\begin{array}{c}f_{0.5-8.0 \mathrm{keV}} \\
\left(10^{-16}[\mathrm{cgs}]\right) \\
(6)\end{array}$ & $\begin{array}{c}L_{0.5-2.0 \mathrm{keV}} \\
(\log \mathrm{cgs}) \\
(7)\end{array}$ & $\begin{array}{c}L_{0.5-8.0 \mathrm{keV}} \\
(\log \mathrm{cgs}) \\
(8)\end{array}$ & $\begin{array}{l}\text { Survey } \\
\quad(9)\end{array}$ \\
\hline CXOECDFS J033122.00-273620.1 & $<11.5$ & $17.0_{-5.8}^{+5.8}$ & $1.8^{\mathrm{a}}$ & $<3.36$ & 8.37 & $<40.2$ & 40.6 & E-CDF-S 02 \\
\hline CXOECDFS J033128.84-275904.8 & $10.9_{-3.6}^{+4.8}$ & $<13.4$ & $1.8^{\mathrm{a}}$ & 2.78 & $<5.71$ & 40.8 & $<41.1$ & E-CDF-S 03 \\
\hline CXOECDFS J033139.05-280221.1 & $1.5_{-1.4}^{+1.4}$ & $<14.6$ & $1.8^{\mathrm{a}}$ & 0.43 & $<7.03$ & 39.9 & $<41.1$ & E-CDF-S 03 \\
\hline CXOECDFS J033143.46-275527.8 & $<4.7$ & $5.4_{-2.5}^{+2.5}$ & $1.8^{\mathrm{a}}$ & $<1.18$ & 2.26 & $<39.6$ & 39.9 & E-CDF-S 03 \\
\hline CXOECDFS J033143.48-275103.0 & $10.2_{-3.8}^{+5.1}$ & $<16.3$ & $1.8^{\mathrm{a}}$ & 2.76 & $<7.41$ & 40.8 & $<41.2$ & E-CDF-S 03 \\
\hline CXOCDFS J033219.10-274445.6 & $5.0_{-2.4}^{+2.4}$ & $<15.6$ & $1.8^{\mathrm{a}}$ & 0.33 & $<1.74$ & 39.6 & $<40.3$ & CDF-S \\
\hline CXOCDFS J033221.91-275427.2 & $6.9_{-3.2}^{+3.2}$ & $<21.5$ & $1.8^{\mathrm{a}}$ & 0.46 & $<2.43$ & 39.6 & $<40.3$ & CDF-S \\
\hline CXOCDFS J033230.01-274404.0 & $87.6_{-10.1}^{+11.2}$ & $104.0_{-11.6}^{+12.8}$ & 1.9 & 6.39 & 12.28 & 40.0 & 40.2 & CDF-S \\
\hline CXOCDFS J033234.73-275533.8 & $<20.6$ & $58.6_{-11.8}^{+11.9}$ & $1.8^{\mathrm{a}}$ & $<1.48$ & 7.05 & $<38.7$ & 39.4 & CDF-S \\
\hline CXOECDFS J033249.26-273610.6 & $38.6_{-6.6}^{+7.8}$ & $56.7_{-8.4}^{+9.8}$ & 1.6 & 9.78 & 27.46 & 40.5 & 40.9 & E-CDF-S 01 \\
\hline CXOECDFS J033316.29-275040.7 & $22.1_{-5.6}^{+6.7}$ & $<22.1$ & $1.8^{\mathrm{a}}$ & 5.90 & $<9.95$ & 40.1 & $<40.3$ & E-CDF-S 04 \\
\hline CXOECDFS J033322.97-273430.7 & $5.3_{-2.5}^{+2.5}$ & $<15.1$ & $1.8^{\mathrm{a}}$ & 1.71 & $<8.17$ & 39.6 & $<40.3$ & E-CDF-S 01 \\
\hline CXOHDFN J123631.66+620907.3 & $34.5_{-7.4}^{+8.5}$ & $40.9_{-9.7}^{+10.9}$ & 1.3 & 1.09 & 2.84 & 39.8 & 40.2 & CDF-N \\
\hline CXOHDFN J123632.55+621039.5 & $<9.5$ & $4.4_{-2.6}^{+2.6}$ & $1.8^{\mathrm{a}}$ & $<0.32$ & 0.25 & $<39.2$ & 39.1 & CDF-N \\
\hline CXOHDFN J123637.18+621135.0 & $17.8_{-4.8}^{+6.0}$ & $21.1_{-6.0}^{+7.2}$ & $1.8^{\mathrm{a}}$ & 0.61 & 1.21 & 39.0 & 39.3 & CDF-N \\
\hline CXOHDFN J123641.81+621132.1 & $33.6_{-6.4}^{+7.5}$ & $39.9_{-7.6}^{+8.8}$ & 1.7 & 1.16 & 2.39 & 39.4 & 39.7 & CDF-N \\
\hline CXOHDFN J123701.47+621845.9 & $44.2_{-8.2}^{+9.3}$ & $50.2_{-10.6}^{+11.7}$ & 1.6 & 1.48 & 3.21 & 40.4 & 40.7 & CDF-N \\
\hline CXOHDFN J123701.99+621122.1 & $19.3_{-5.1}^{+6.2}$ & $21.9_{-6.2}^{+7.6}$ & $1.8^{\mathrm{a}}$ & 0.60 & 1.15 & 39.5 & 39.7 & CDF-N \\
\hline CXOHDFN J123706.12+621711.9 & $17.6_{-5.7}^{+6.8}$ & $34.0_{-8.3}^{+9.6}$ & $1.8^{\mathrm{a}}$ & 0.55 & 1.80 & 40.0 & 40.5 & CDF-N \\
\hline CXOHDFN J123715.94+621158.3 & $\begin{array}{l}17.7_{-5.2}^{+6.3} \\
\end{array}$ & $20.3_{-6.6}^{+7.8}$ & $1.8^{\mathrm{a}}$ & 0.62 & 1.21 & 39.2 & 39.5 & $\mathrm{CDF}-\mathrm{N}$ \\
\hline CXOHDFN J123721.60+621246.8 & $18.6_{-5.4}^{+6.2}$ & $29.8_{-7.6}^{+8.0}$ & $1.8^{\mathrm{a}}$ & 0.77 & 2.07 & 39.3 & 39.8 & $\mathrm{CDF}-\mathrm{N}$ \\
\hline CXOHDFN J123723.45+621047.9 & $21.0_{-6.1}^{+7.4}$ & $<27.7$ & $1.8^{\mathrm{a}}$ & 0.72 & $<1.60$ & 39.4 & $<39.7$ & CDF-N \\
\hline CXOHDFN J123727.71+621034.3 & $32.5_{-7.5}^{+8.1}$ & $35.9_{-10.0}^{+11.2}$ & 1.1 & 1.07 & 2.94 & 40.1 & 40.6 & CDF-N \\
\hline CXOHDFN J123730.60+620943.1 & $34.5_{-7.9}^{+9.5}$ & $67.2_{-12.1}^{+13.2}$ & 0.9 & 1.15 & 6.37 & 40.5 & 41.2 & CDF-N \\
\hline Median Values ${ }^{b}$ & 19.3 & 35.9 & $1.8^{\mathrm{c}}$ & 1.09 & 2.84 & 39.9 & 40.2 & $\ldots$ \\
\hline
\end{tabular}

${ }^{a}$ Indicates this $\Gamma$ value was assigned; see $\S 2.2$ for details.

${ }^{\mathrm{b}}$ Quoted median values were computed using only the sources which were detected in a given band (i.e., limits were not included). 
${ }^{\mathrm{c}}$ Indicates mean value determined from stacking analyses (see $\S 2.2$ for details).

Note. - All fluxes and luminosities have been corrected for Galactic absorption as discussed at the end of $\S 1$. Col.(1) Off-nuclear X-ray source name. Col.(2) 0.5-2.0 keV source counts. Col.(3) $0.5-8.0 \mathrm{keV}$ source counts. Col.(4) Effective photon index $\Gamma$. When the number of counts is low (i.e., $<30$ counts in the $0.5-2.0 \mathrm{keV}$ band), we set $\Gamma=1.8$ as per the discussion in $\S 2.2$. Other values of $\Gamma$ are as reported by Alexander et al. (2003; CDF-N and CDF-S) or Lehmer et al. (2005; E-CDF-S). Col.(5) 0.5-2.0 keV flux in units of $10^{-16} \mathrm{erg} \mathrm{cm}^{-2} \mathrm{~s}^{-1}$. Col.(6) 0.5-8.0 keV flux in units of $10^{-16} \mathrm{erg} \mathrm{cm}^{-2} \mathrm{~s}^{-1}$. Col.(7) Logarithm of the $0.5-2.0 \mathrm{keV}$ luminosity in units of erg s ${ }^{-1}$. Col.(8) Logarithm of the 0.5-8.0 keV luminosity in units of erg s ${ }^{-1}$. Col.(9) Survey field in which each source was identified. For E-CDF-S identifications, the associated field number (i.e., 01-04) indicates the Chandra pointing within which the source was detected (see Lehmer et al. 2005 for details). 
Table 2. Off-Nuclear Sources: Additional Properties

\begin{tabular}{|c|c|c|c|c|c|c|c|c|c|}
\hline $\begin{array}{c}\text { Source Name } \\
\text { (1) }\end{array}$ & $\begin{array}{l}\text { Positional } \\
\text { (arcsec) } \\
(2)\end{array}$ & $\begin{array}{l}\text { Offset In Units } \\
\text { (pos. err.) } \\
\text { (3) }\end{array}$ & $\begin{array}{c}\text { s Of. . } \\
(\mathrm{kpc}) \\
(4)\end{array}$ & $\begin{array}{c}V_{606} \\
(\mathrm{mag}) \\
(5)\end{array}$ & $\begin{array}{c}z \\
(6)\end{array}$ & $\begin{array}{c}M_{606} \\
(\mathrm{mag}) \\
(7)\end{array}$ & $\begin{array}{c}\nu L_{\nu}(6000 \AA) \\
(\log \mathrm{cgs}) \\
(8)\end{array}$ & $\begin{array}{c}\text { H04 Detection? } \\
(\mathrm{Y} / \mathrm{N}) \\
(9)\end{array}$ & $\begin{array}{c}\text { Optical Knot? } \\
(\mathrm{Y} / \mathrm{N}) \\
(10)\end{array}$ \\
\hline CXOECDFS J033122.00-273620.1 & 3.60 & 2.11 & 8.88 & 18.80 & $0.140^{\mathrm{P}}$ & -20.1 & 43.4 & $\mathrm{~N}$ & $\mathrm{~N}$ \\
\hline CXOECDFS J033128.84-275904.8 & 1.70 & 2.00 & 6.97 & 19.22 & $0.266^{\mathrm{p}}$ & -21.4 & 43.9 & $\mathrm{~N}$ & $\mathrm{~N}$ \\
\hline CXOECDFS J033139.05-280221.1 & 2.28 & 2.25 & 8.96 & 18.24 & $0.251^{\mathrm{P}}$ & -22.2 & 44.2 & $\mathrm{~N}$ & $\mathrm{~N}$ \\
\hline CXOECDFS J033143.46-275527.8 & 1.71 & 2.02 & 3.49 & 17.94 & $0.112^{\mathrm{P}}$ & -20.4 & 43.5 & $\mathrm{~N}$ & $\mathrm{~N}$ \\
\hline CXOECDFS J033143.48-275103.0 & 2.28 & 1.91 & 9.28 & 19.57 & $0.265^{\mathrm{p}}$ & -21.1 & 43.8 & $\mathrm{~N}$ & $\mathrm{~N}$ \\
\hline CXOCDFS J033219.10-274445.6 & 1.21 & 2.02 & 4.01 & 20.84 & $0.201^{\mathrm{P}}$ & -19.0 & 43.0 & $\mathrm{~N}$ & $\mathrm{~N}$ \\
\hline CXOCDFS J033221.91-275427.2 & 1.81 & 2.37 & 5.58 & 19.82 & $0.184^{\mathrm{P}}$ & -19.9 & 43.3 & $\mathrm{~N}$ & $\mathrm{~N}$ \\
\hline CXOCDFS J033230.01-274404.0 & 0.91 & 1.52 & 1.31 & 17.53 & $0.076^{\mathrm{s}}$ & -20.2 & 43.4 & $\mathrm{Y}$ & $\mathrm{Y}$ \\
\hline CXOCDFS J033234.73-275533.8 & 4.71 & 5.24 & 3.55 & 16.78 & $0.038^{\mathrm{s}}$ & -19.4 & 43.1 & $\mathrm{Y}$ & $\mathrm{N}$ \\
\hline CXOECDFS J033249.26-273610.6 & 3.30 & 5.03 & 6.72 & 17.90 & $0.112^{\mathrm{p}}$ & -18.9 & 42.9 & $\mathrm{~N}$ & $\mathrm{Y}$ \\
\hline CXOECDFS J033316.29-275040.7 & 3.24 & 2.31 & 5.47 & 17.84 & $0.090^{\mathrm{P}}$ & -20.4 & 43.5 & $\mathrm{~N}$ & $\mathrm{~N}$ \\
\hline CXOECDFS J033322.97-273430.7 & 2.30 & 1.51 & 4.08 & 19.20 & $0.096^{\mathrm{p}}$ & -18.9 & 42.9 & $\mathrm{~N}$ & $\mathrm{~N}$ \\
\hline CXOHDFN J123631.66+620907.3 & 1.26 & 2.04 & 3.29 & 20.15 & $0.150^{\mathrm{P}}$ & -19.1 & 43.0 & $\mathrm{~N}$ & $\mathrm{Y}$ \\
\hline CXOHDFN J123632.55+621039.5 & 2.02 & 3.36 & 4.86 & 20.82 & $0.136^{\mathrm{s}}$ & -18.2 & 42.7 & $\mathrm{~N}$ & $\mathrm{Y}$ \\
\hline CXOHDFN J123637.18+621135.0 & 2.23 & 3.71 & 3.31 & 18.39 & $0.079^{\mathrm{s}}$ & -19.3 & 43.1 & $\mathrm{Y}$ & $\mathrm{Y}$ \\
\hline CXOHDFN J123641.81+621132.1 & 1.23 & 2.04 & 2.04 & 20.08 & $0.089^{\mathrm{s}}$ & -17.9 & 42.6 & $\mathrm{Y}$ & $\mathrm{Y}$ \\
\hline CXOHDFN J123701.47+621845.9 & 2.89 & 4.65 & 10.69 & 18.98 & $0.232^{\mathrm{s}}$ & -21.3 & 43.9 & $\mathrm{Y}$ & $\mathrm{N}$ \\
\hline CXOHDFN J123701.99+621122.1 & 1.24 & 2.07 & 2.98 & 19.45 & $0.136^{\mathrm{s}}$ & -19.6 & 43.2 & Y & $\mathrm{Y}$ \\
\hline CXOHDFN J123706.12+621711.9 & 1.01 & 1.68 & 3.98 & 19.07 & $0.254^{\mathrm{s}}$ & -21.4 & 44.0 & $\mathrm{~N}$ & $\mathrm{Y}$ \\
\hline CXOHDFN J123715.94+621158.3 & 1.11 & 1.84 & 2.13 & 18.70 & $0.105^{\mathrm{s}}$ & -19.7 & 43.3 & Y & $\mathrm{N}$ \\
\hline CXOHDFN J123721.60+621246.8 & 2.62 & 4.36 & 5.07 & 18.15 & $0.106^{\mathrm{s}}$ & -20.3 & 43.5 & $\mathrm{Y}$ & $\mathrm{Y}$ \\
\hline CXOHDFN J123723.45+621047.9 & 2.87 & 4.31 & 5.90 & 19.56 & $0.113^{\mathrm{s}}$ & -19.0 & 43.0 & $\mathrm{Y}$ & $\mathrm{N}$ \\
\hline CXOHDFN J123727.71+621034.3 & 2.05 & 2.73 & 7.14 & 18.51 & $0.214^{\mathrm{s}}$ & -21.5 & 44.0 & $\mathrm{~N}$ & $\mathrm{~N}$ \\
\hline CXOHDFN J123730.60+620943.1 & 1.48 & 1.68 & 6.54 & 19.48 & $0.298^{\mathrm{s}}$ & -21.5 & 44.0 & $\mathrm{~N}$ & $\mathrm{Y}$ \\
\hline Median Values & 2.05 & 2.11 & 5.07 & 19.07 & 0.14 & -20.2 & 43.4 & $\ldots$ & $\ldots$ \\
\hline
\end{tabular}

Note. - Col.(1) Off-nuclear X-ray source name. Col.(2) X-ray positional offset from the associated optical-source nucleus in units of arcseconds. Col.(3) Same offset in previous column in units of the Chandra positional error. Col.(4) Physical offset in units of kpc. Col.(5) ACS V606 observed magnitude of host 
galaxy. Col.(6) Redshift estimate of the host galaxy for each off-nuclear X-ray source. Superscripts "s" and "p" indicate spectroscopic and photometric redshifts, respectively (see $\S 2$ for details). Col.(7) Absolute magnitude of the host galaxy $\left(M_{606}\right)$ at $\lambda \approx 6000 \AA$. Col. (8) Logarithm of the host-galaxy, rest-frame 6000 $\AA$ luminosity in units of $\mathrm{erg} \mathrm{s}^{-1}$. Col.(9) Indicates whether each source was previously detected by H04. Col.(10) Indicates whether an optical knot is observed

to be coincident with the X-ray source position. 


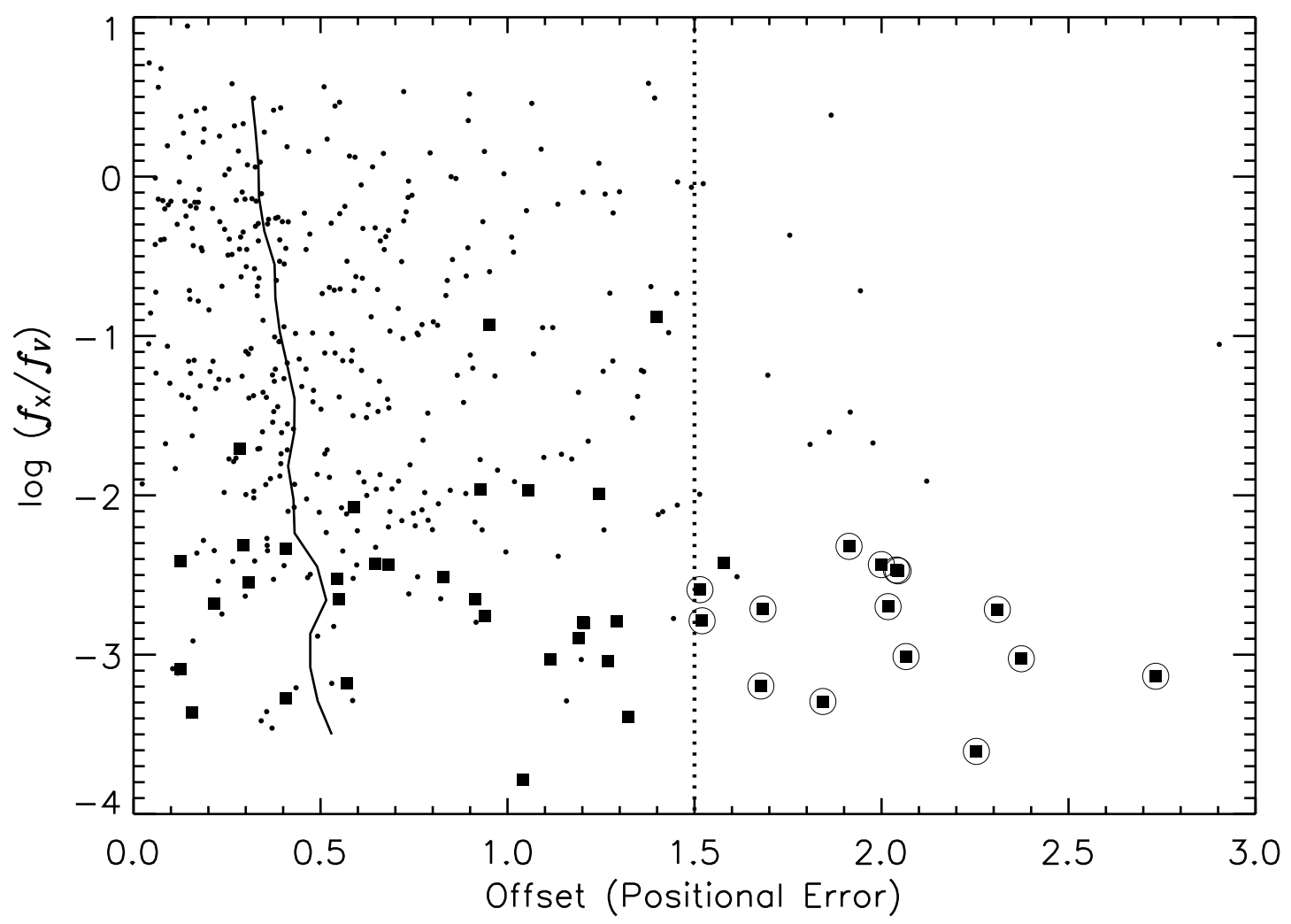

Fig. 1. - Logarithm of the X-ray-to-optical flux ratio vs. offset in units of positional error for 0.5-2.0 keV detected sources matched to optical sources with $V_{606}<24$. Large filled squares indicate sources with $V_{606}<21$, and the circled sources are our off-nuclear source candidates. Flux ratios were computed using point-source X-ray fluxes and integrated host-galaxy optical fluxes. The vertical dotted line indicates the location of $1.5 \times$ the Chandra positional error, which was used to distinguish sources as off-nuclear (see $\S 2.1$ ). Sources with larger $\log \left[f_{\mathrm{X}} / f_{\mathrm{V}}\right]$ that lie outside $1.5 \times$ the Chandra positional error are generally low-significance matches and lie outside the optical extent of the matched source. The solid curve indicates the running median offset for sources within a region of size $\Delta \log \left[f_{\mathrm{X}} / f_{\mathrm{V}}\right]=1.5$. The general trend of improved median positional accuracy with increasing $\log \left[f_{\mathrm{X}} / f_{\mathrm{V}}\right]$ is expected as the number of "point-like" AGNs contributing to the statistics continually increases. 


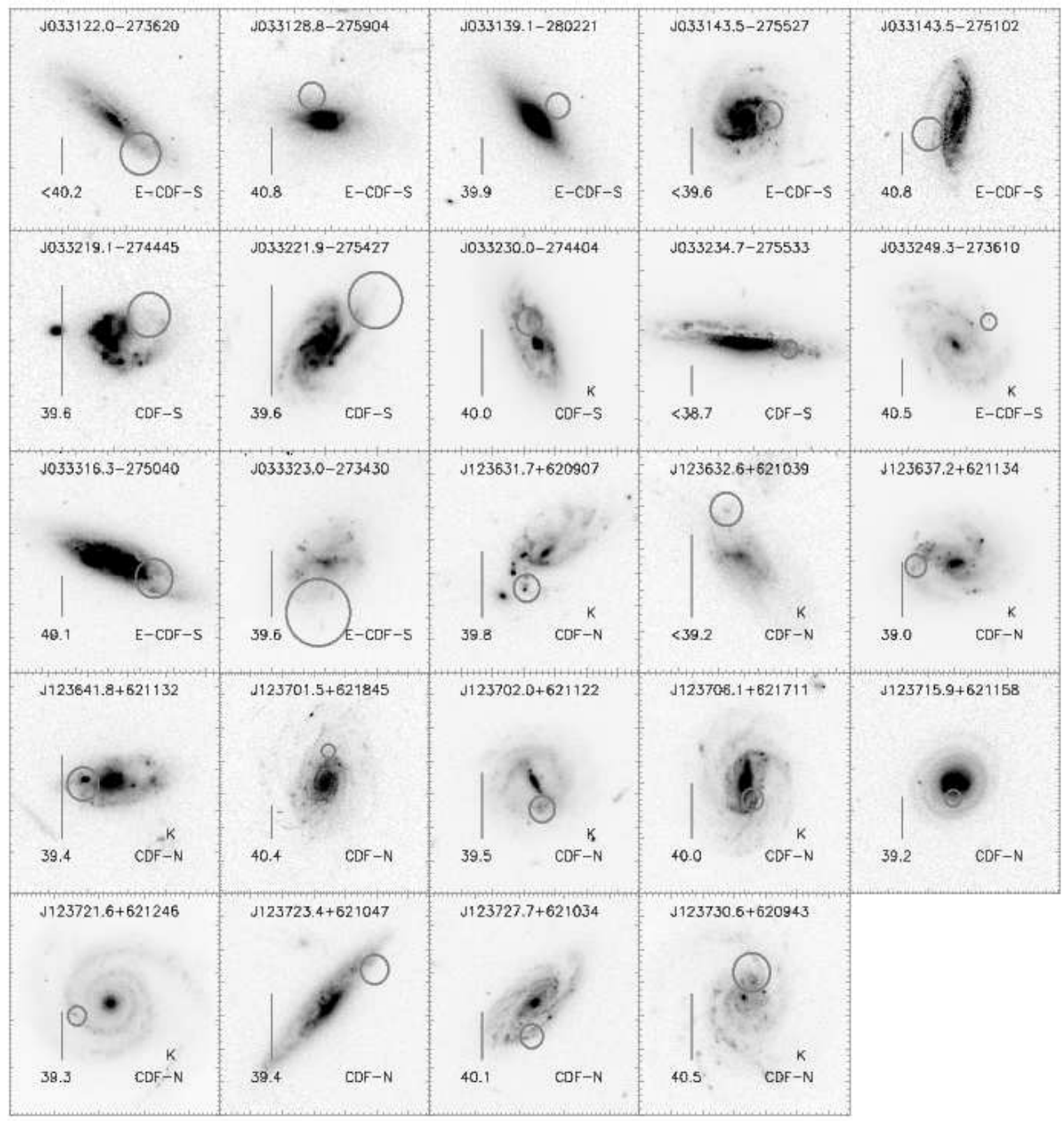

Fig. 2.- Advanced Camera for Surveys (ACS) $V_{606}$-band postage-stamp images of each off-nuclear source host galaxy. In each image, we show the off-nuclear source name $(t o p)$, the survey field in which the source is detected (lower right), and the logarithm of the $0.5-2.0 \mathrm{keV}$ luminosity in $\mathrm{erg} \mathrm{s}^{-1}$ (lower left); a "K" is displayed above the survey field if the off-nuclear source is visually coincident with an optical knot. All sources are detected in either the $0.5-2.0 \mathrm{keV}$ or $0.5-8.0 \mathrm{keV}$ bands; the upper limits shown here are for sources not detected in the $0.5-2.0 \mathrm{keV}$ band. For illustrative purposes, the images are not all the same size; the scale of each image can be deduced from the vertical $3^{\prime \prime}$ bar located in the lower-left corner of each frame. Each off-nuclear source is marked with a circle having a radius equal to the Chandra $80-90 \%$ positional error. 


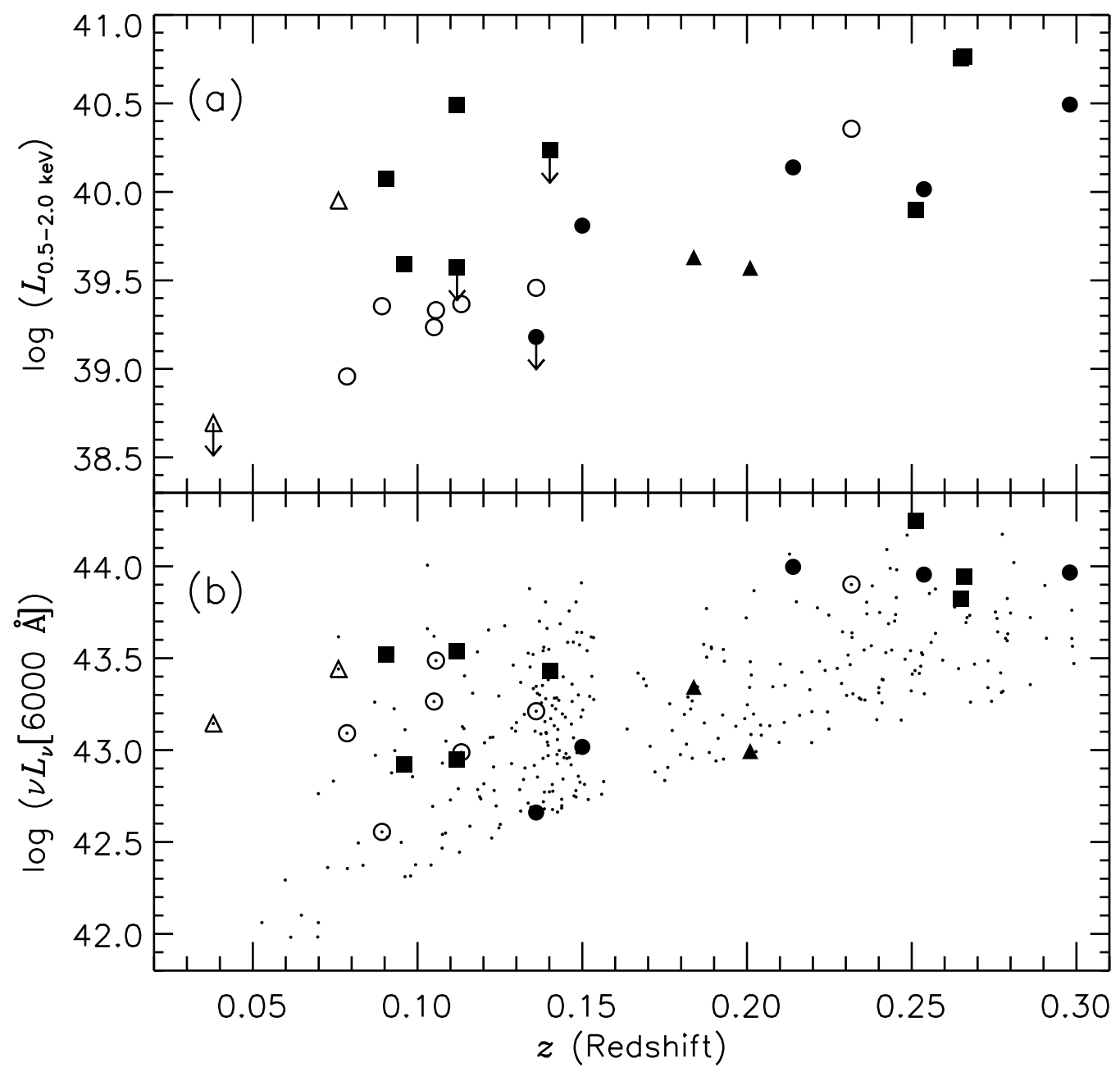

Fig. 3.- (a) X-ray luminosity and redshift of each off-nuclear source. Symbols correspond to objects detected in the CDF-N (circles), CDF-S (triangles), and E-CDF-S (squares) surveys. Filled symbols correspond to off-nuclear sources unique to this study (i.e., not previously discovered by Hornschemeier et al. 2004). Upper limits correspond to sources detected in the $0.5-8.0 \mathrm{keV}$ band but not in the $0.5-2.0 \mathrm{keV}$ band. (b) Rest-frame $6000 \AA$ optical luminosity for spiral and irregular galaxies with $V_{606}<21$ as a function of redshift (small filled circles). Galaxies hosting an off-nuclear source have been outlined with symbols following the convention of Figure 3a. 


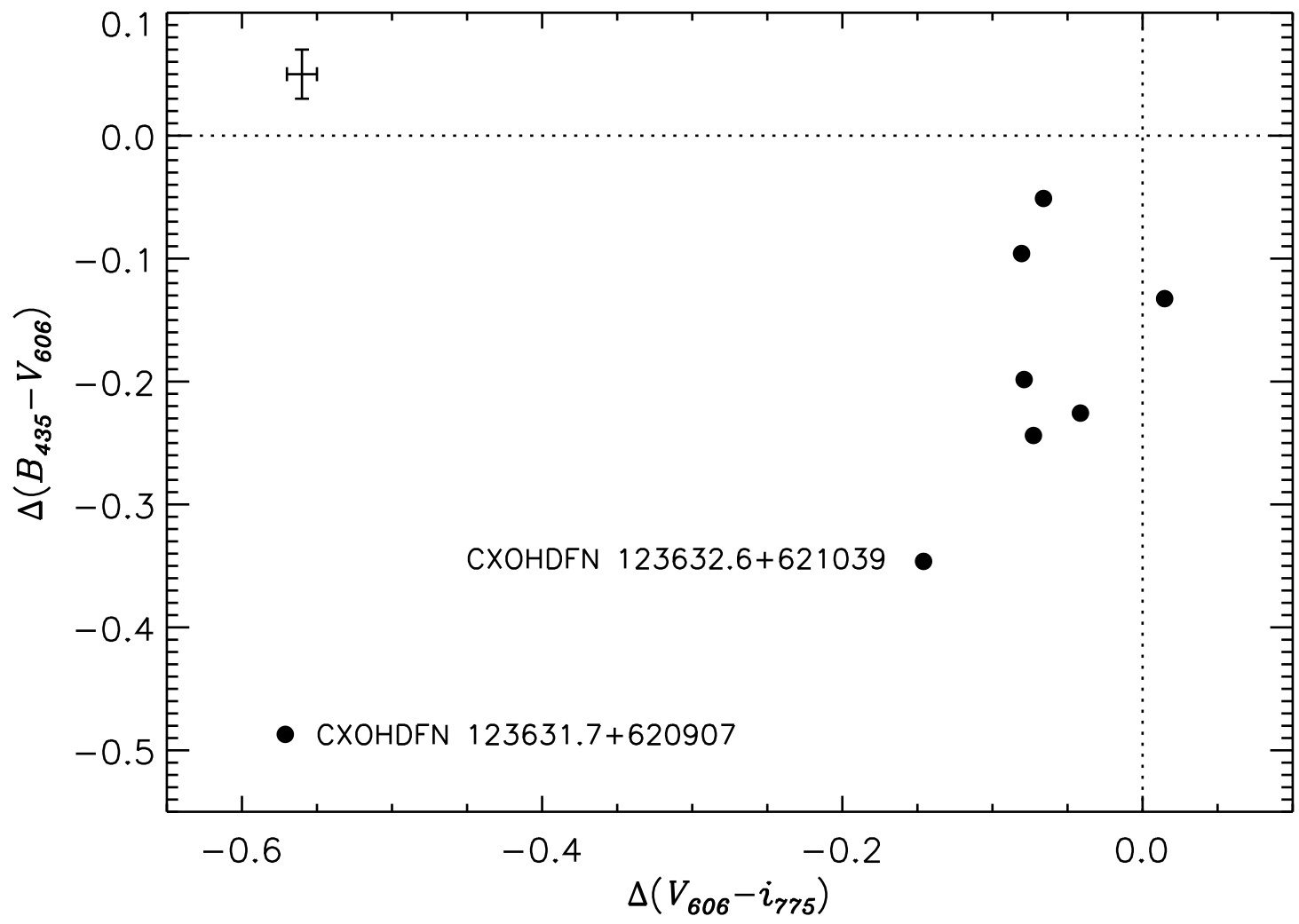

Fig. 4.- Relative color difference between optical knot and host galaxy $\left(\Delta\left(B_{435}-V_{606}\right)\right.$ vs. $\Delta\left(V_{606}-i_{775}\right)$; see $\left.\S 2.2\right)$ for the eight optical knots coincident with off-nuclear X-ray sources in the GOODS regions (filled circles). The plotted error bar in the upper-left corner shows the typical errors of these measurements; the dotted lines show the expected values for the case of the optical knots having the same colors as their host galaxies. The relatively blue optical-knot colors show that these off-nuclear regions are likely populated by a younger stellar population; the source names for the two most extreme cases have been noted. 


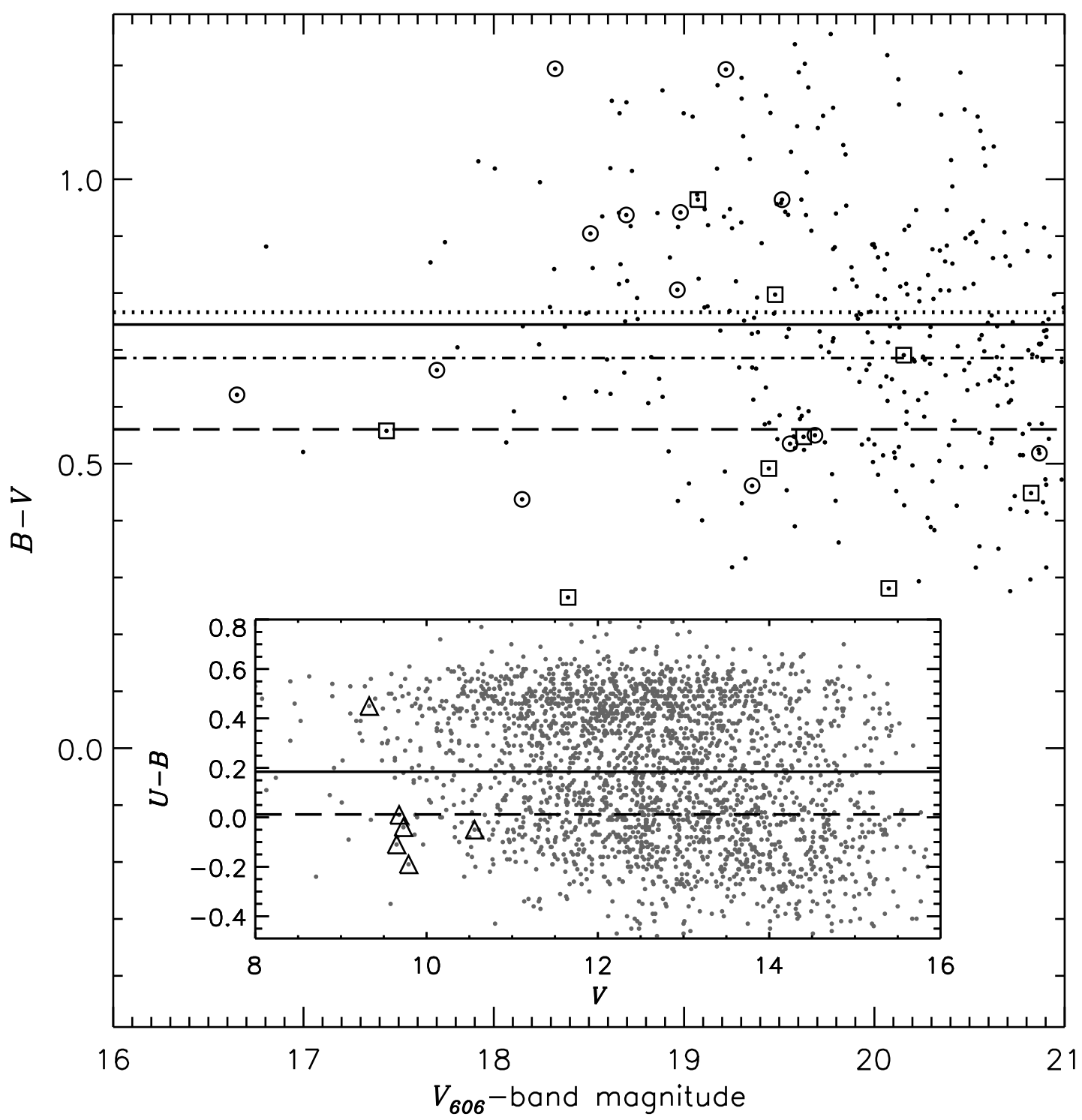

Fig. 5.- Optical $B-V$ color vs. $V_{606}$ magnitude for $V_{606}<21, z<0.3$ field galaxies in the CDFs (small filled circles). Open symbols indicate galaxies hosting off-nuclear X-ray sources with (squares) and without (circles) optical knots. The horizontal lines indicate mean $B-V$ values for field galaxies (solid line), galaxies hosting off-nuclear sources (dot-dashed line), and galaxies hosting off-nuclear sources with (dashed line) and without (dotted line) optical knots. The inset plot shows the optical colors (i.e., Johnson $U-B$ vs. $V$ ) for local galaxies from the RC3 catalog (gray filled circles). The six sources highlighted by open triangles are galaxies hosting IXOs coincident with luminous optical knots, and the horizontal lines represent the mean $U-B$ values for RC3 galaxies (solid line) and the subset of RC3 IXOhosting galaxies with optical knots (dashed line). Note that for both samples, the optical knots are preferentially located in relatively blue, star-forming galaxies. 


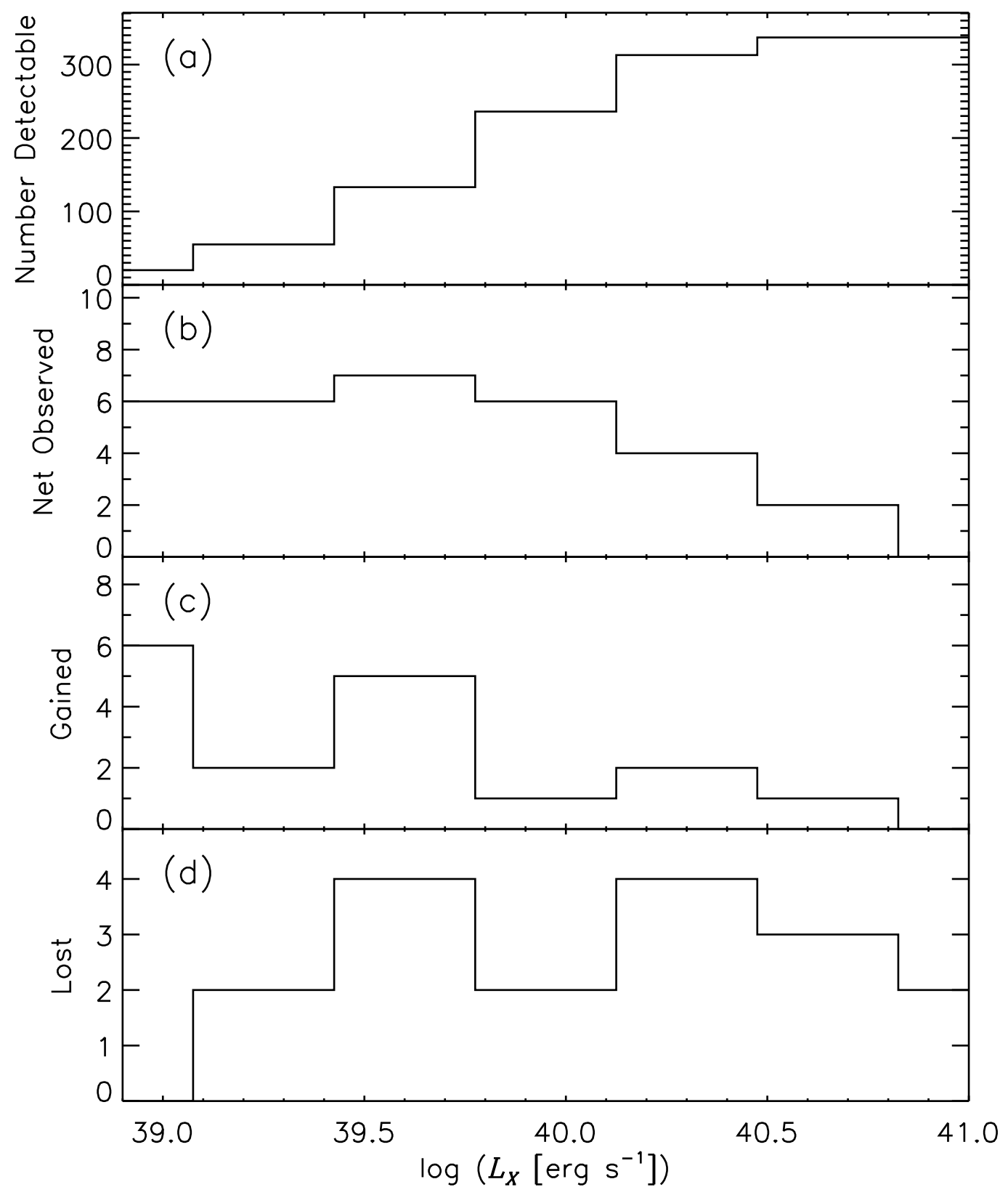

Fig. 6.- (a) Number of galaxies in the Chandra deep fields in which we could detect an off-nuclear source of $0.5-2.0 \mathrm{keV}$ luminosity $L_{\mathrm{X}}$. The highest $L_{\mathrm{X}}$ bin contains all 337 spiral and irregular galaxies in our sample. (b) Number of galaxies in each $L_{\mathrm{X}}$ bin of panel (a) containing an off-nuclear source with an X-ray luminosity of $L_{\mathrm{X}}$ or greater. (c) Number of galaxies gained in each $L_{\mathrm{X}}$ bin of panel (b) progressing from the lowest $L_{\mathrm{X}}$ bin to the highest. (d) Number of galaxies dropping out of each $L_{\mathrm{X}}$ bin of panel (b) progressing from the lowest $L_{\mathrm{X}}$ bin to the highest. 


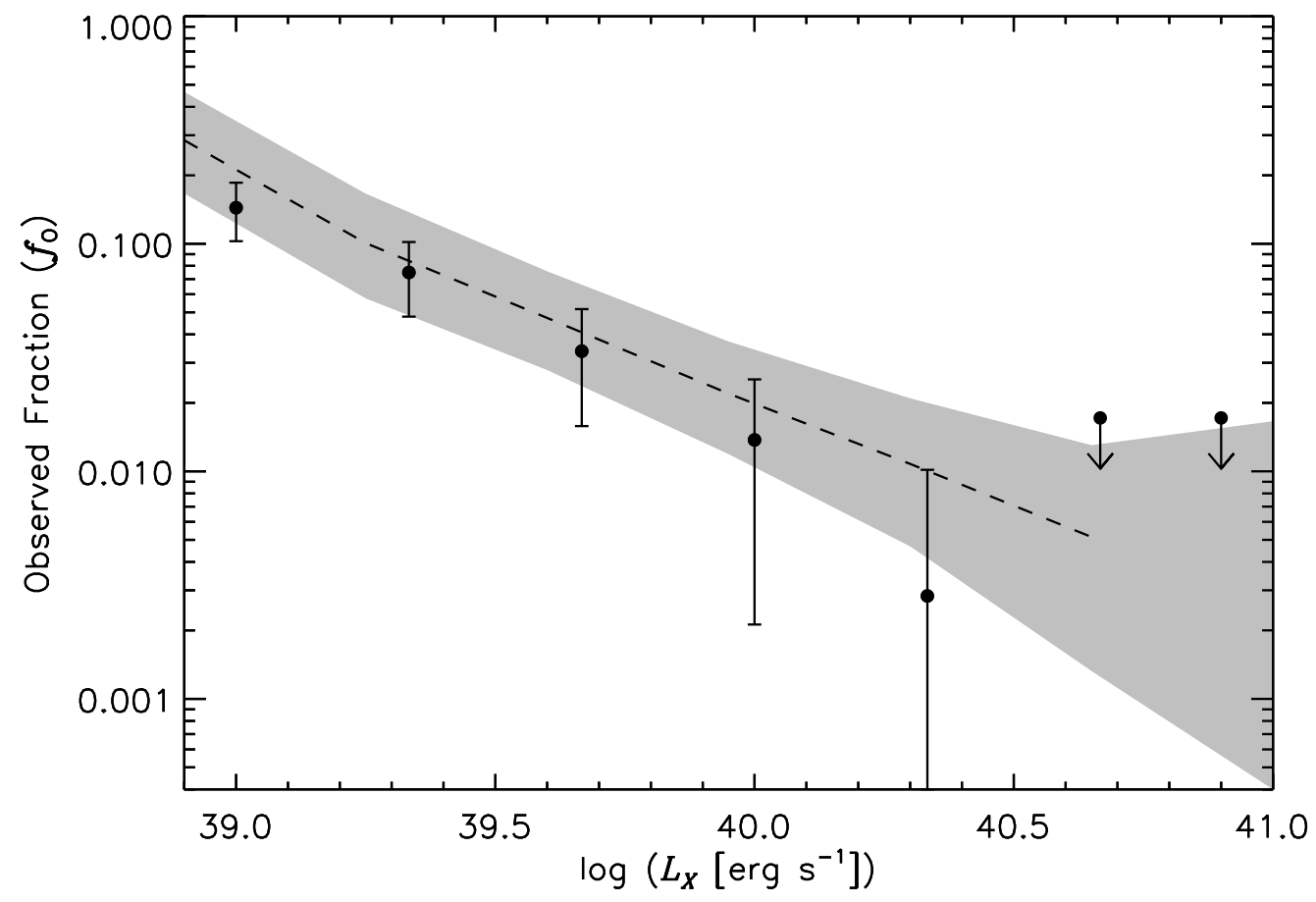

Fig. 7.- Observed fraction of galaxies in the Chandra deep fields hosting an off-nuclear source with a $0.5-2.0 \mathrm{keV}$ luminosity of $L_{\mathrm{X}}$ or greater (dashed line). The shaded area shows the $1 \sigma$ confidence region, computed using the methods outlined in Gehrels (1986). The dashed line terminates for $L_{\mathrm{X}} \gtrsim 10^{40.7} \mathrm{erg} \mathrm{s}^{-1}$ due to the lack of off-nuclear sources with luminosities in this regime; the shaded region here represents the $3 \sigma$ upper limit. The solid points with $1 \sigma$ error bars and $3 \sigma$ upper limits represent the observed fraction for the matched PC04 subsample of local galaxies. 


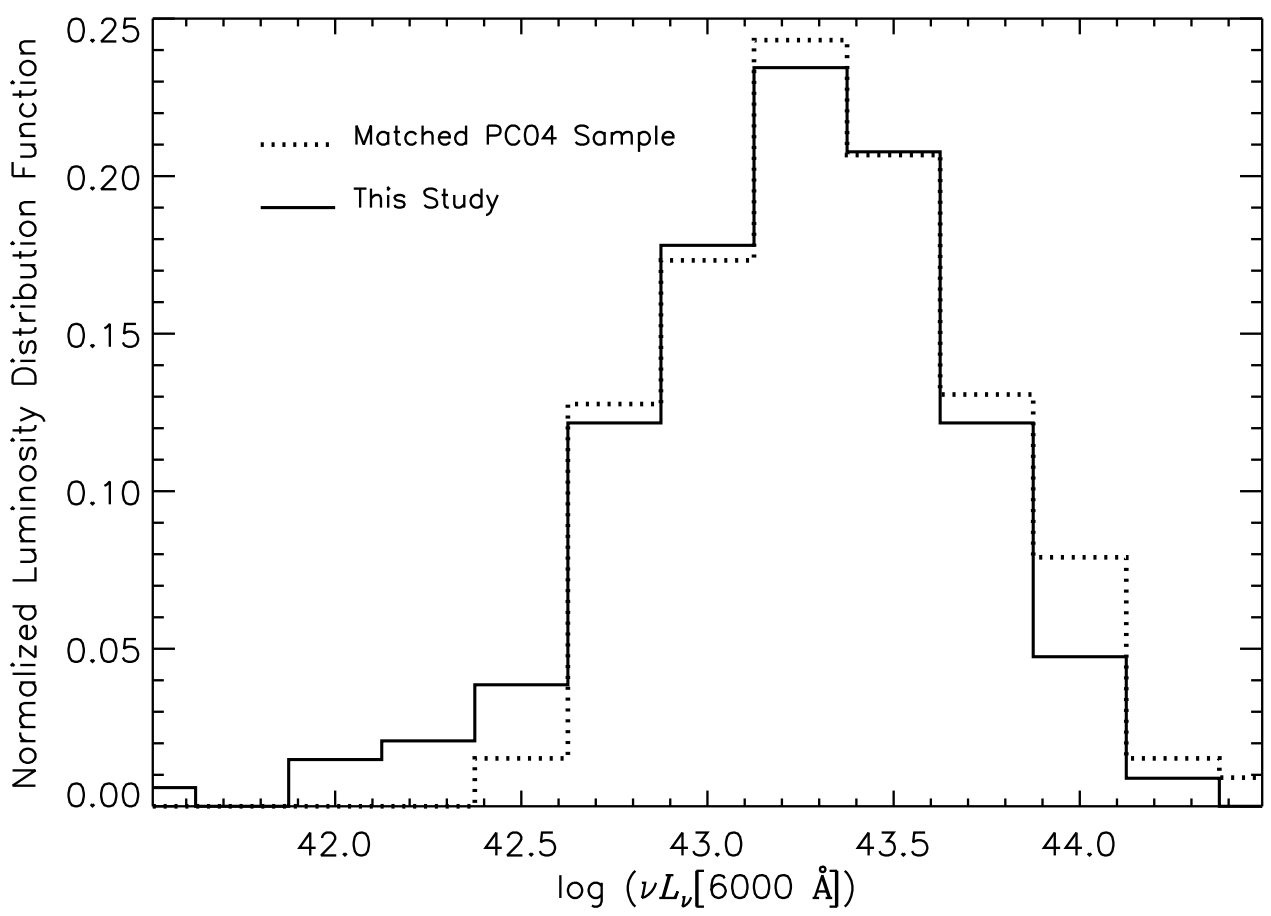

Fig. 8.- Normalized optical-luminosity distributions for field galaxies in this survey (solid histogram) and a matched sample of galaxies in the local universe adapted from the PC04 sample (dotted histogram). To construct the matched sample, we adjusted the original PC04 galaxy sample to include only galaxies with luminosities $\nu L_{\nu}(6000 \AA) \gtrsim 10^{42.6} \mathrm{erg} \mathrm{s}^{-1}$; this is equivalent to requiring $V_{606}<21$ for these galaxies if they were placed at $z=0.14$. 


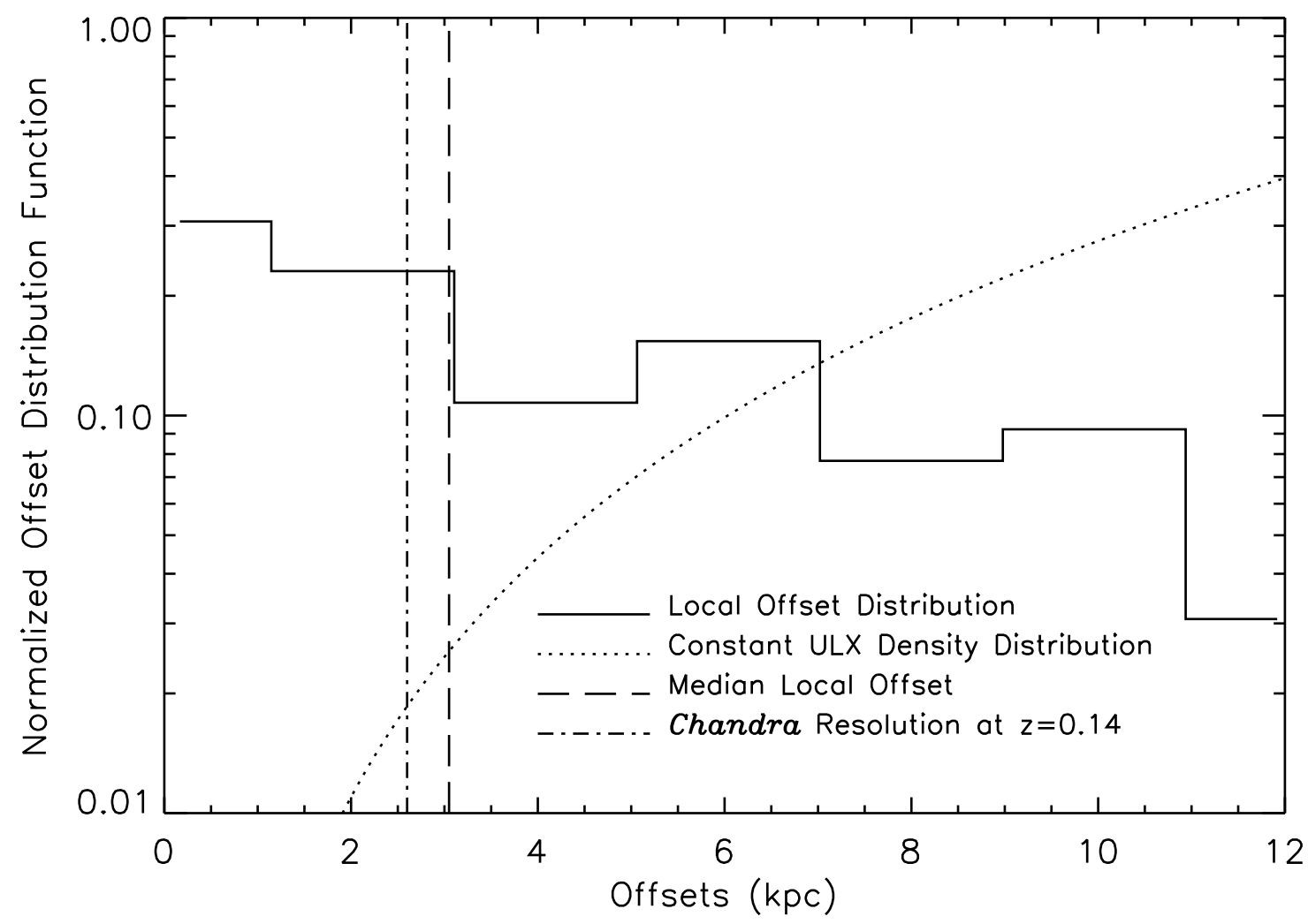

Fig. 9.- Projected physical offset distribution function of Chandra-observed local IXOs (solid histogram). The dashed vertical line represents the median offset for the IXOs used to create the offset distribution function. The dot-dashed vertical line indicates the approximate resolution of Chandra at $z=0.14$, the median redshift of our intermediate-redshift, offnuclear X-ray source sample. The dotted curve shows the expected distribution of offsets for a constant-density galactic distribution of off-nuclear X-ray sources. 


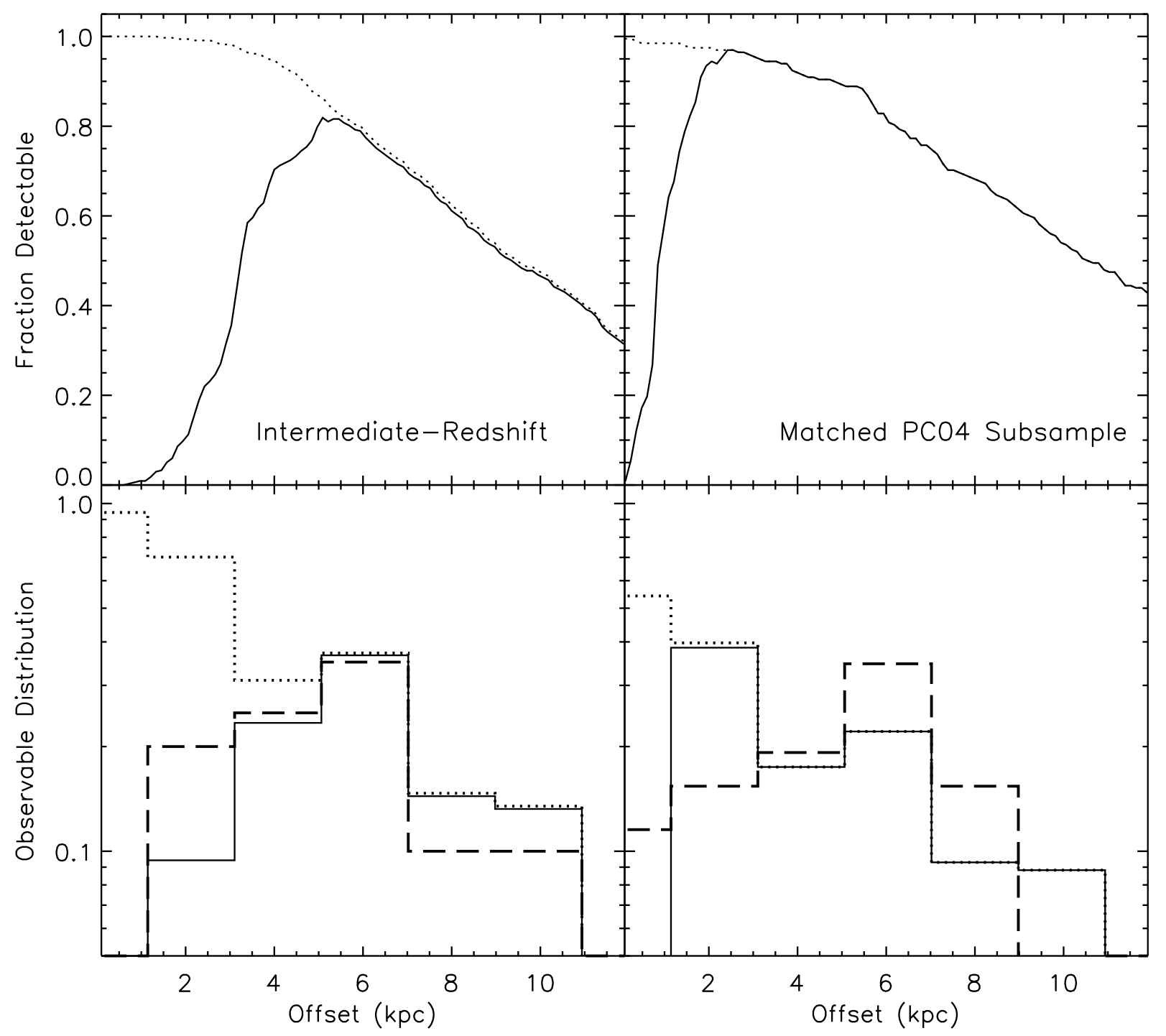

Fig. 10.- (top panels) Fraction of galaxies in which an off-nuclear source could have been detected at a given offset for our data (top-left panel) and for IXOs in the PC04 sample assuming a galaxy size of $0.5 \times$ the RC3 major-axis diameter, $D_{25}$ (top-right panel). Solid lines correspond to the actual resolution of the observations, and dotted lines correspond to the case where there is no resolution limit. (bottom panels) The solid and dotted histograms show the expected distributions of off-nuclear sources given the respective resolution constraints in the top panels and the local Chandra-observed IXO distribution (i.e., Figure 9); the left and right panels correspond to our data and the PC04 data, respectively. For comparison, the actual measured distributions have been plotted (dashed histograms). 


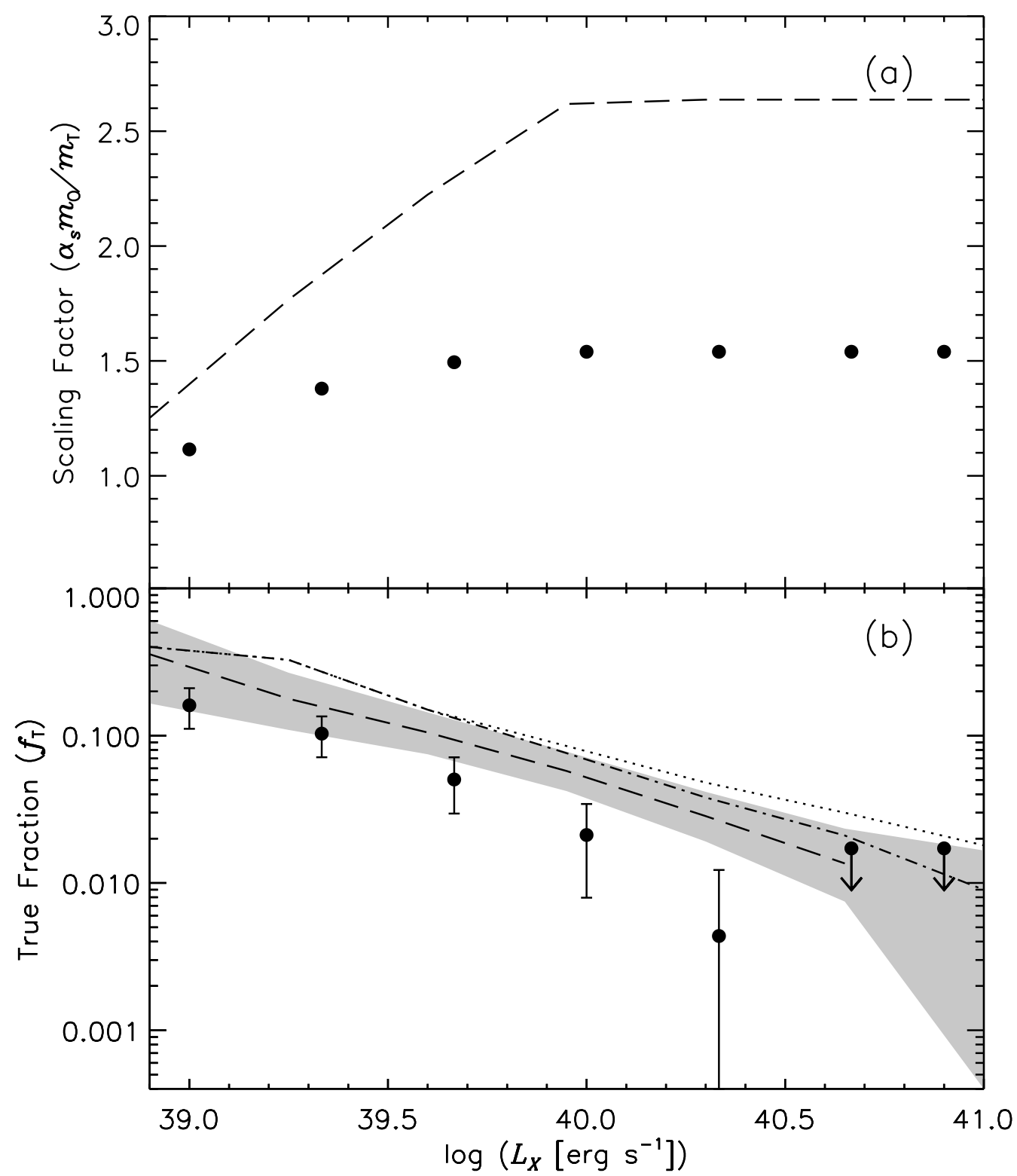

Fig. 11.- (a) Scaling factor $\left(\alpha_{\mathrm{s}} m_{\mathrm{O}} / m_{\mathrm{T}}\right)$ applied to the observed fraction $\left(f_{\mathrm{O}}\right.$; Figure 7$)$ to obtain the true fraction $\left(f_{\mathrm{T}}\right.$; Figure 11b) for our sample (dashed line) and the matched PC04 subsample (filled circles). These factors were obtained using the methods discussed in $\S 3$. (b) True fraction $\left(f_{\mathrm{T}}\right)$ of galaxies in the Chandra deep fields hosting an off-nuclear source with a 0.5-2.0 keV luminosity of $L_{\mathrm{X}}$ or greater (dashed line with shaded $1 \sigma$ confidence region). The filled circles with error bars represent the equivalent true fraction for the matched PC04 subsample of local galaxies. In the region where $L_{\mathrm{X}} \gtrsim 10^{40.7} \mathrm{erg} \mathrm{s}^{-1}$, there are no off-nuclear sources in either sample (i.e., the intermediate-redshift or PC04 subsample), and therefore we have plotted $3 \sigma$ upper limits. The dotted line shows the total X-ray detection fraction for the 337 spiral and irregular galaxies in our sample, which provides an upper limit to the true fraction of galaxies hosting off- 


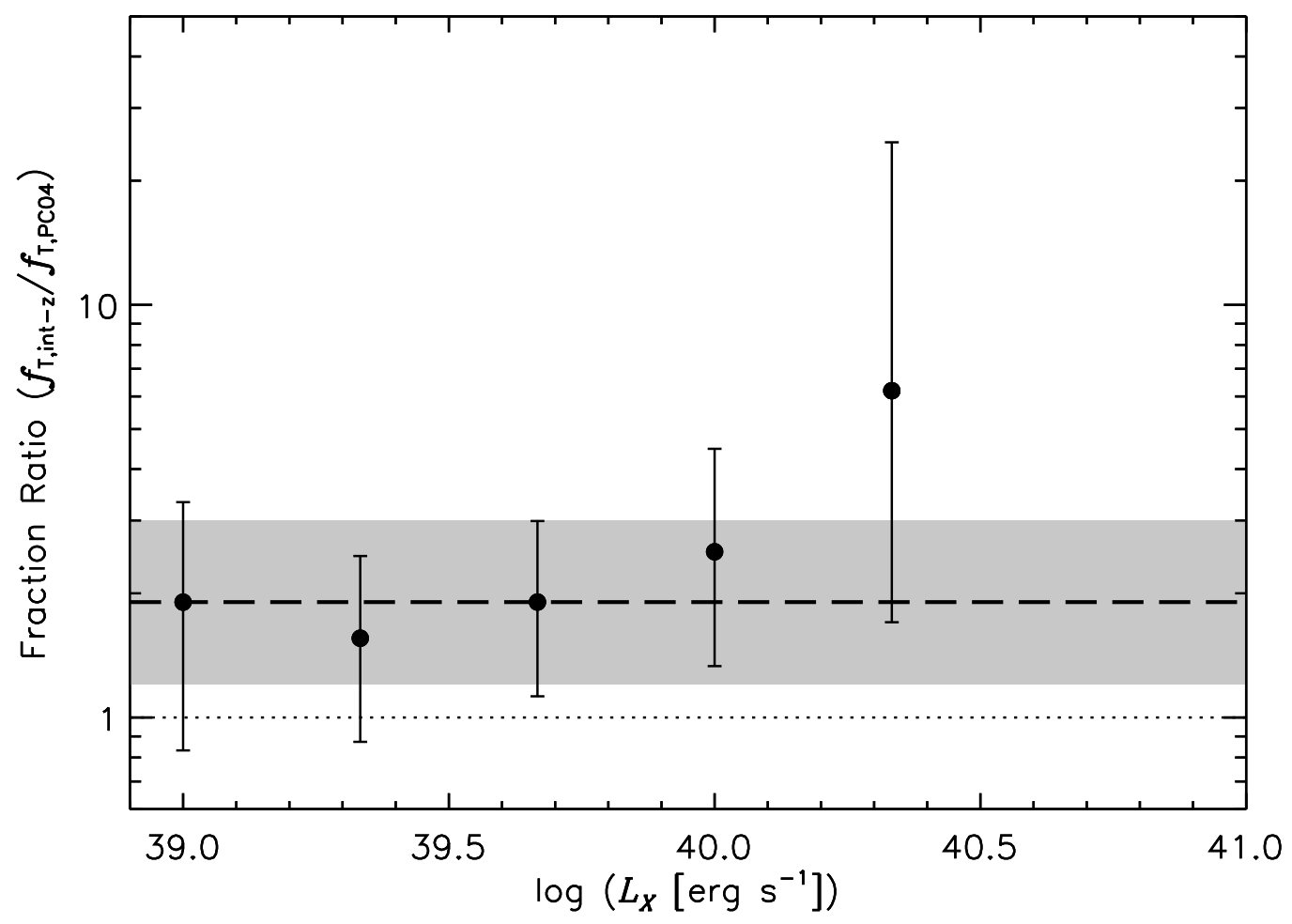

Fig. 12.- Ratios of off-nuclear source incidence fractions of our intermediate-redshift galaxy sample and the matched local PC04 subsample $\left(f_{\mathrm{T}, \mathrm{int}-z} / f_{\mathrm{T}, \mathrm{PC} 04}\right.$; filled circles with $1 \sigma$ error bars); the median ratio is shown as a horizontal dashed line. The shaded region shows the expected increase in the fraction at $z \approx 0.05-0.3$ due to the global increase in star-formation density at these redshifts and the dotted horizontal line shows the expected ratio for the case of no evolution. 